\title{
ON A SYSTEM COUPLING TWO-CRYSTALLIZATION ALLEN-CAHN EQUATIONS AND A SINGULAR NAVIER-STOKES SYSTEM*
}

\author{
BIANCA MORELLI RODOLFO CALSAVARA ${ }^{\dagger}$ AND JOSÉ LUIZ BOLDRINI ${ }^{\ddagger}$
}

\begin{abstract}
We present a result on existence of solutions for a system of highly nonlinear and singular partial differential equations obtained by coupling the two-crystallization Allen-Cahn equations to a singular Navier-Stokes system and a nonlinear heat equation.

Such a system constitutes a phase field model for non-isothermal solidification/melting processes of certain metallic alloys for which two different kinds of crystallization are possible. In this model, the liquid phase and each one of the possible crystallization states are described by their own phase fields. The possibility of occurrence of fluid flow in a a priori unknown non-solid region is also considered, turning the model into a free-boundary value problem.
\end{abstract}

Key words. Solidification, phase field, convection, free-boundary value problem, parabolic partial differential equations, fixed point.

AMS subject classifications. 35K40, 80A22, 47H10, 76R10.

\section{Introduction}

We will analyze a system of nonlinear partial differential equations subjected to boundary and initial conditions, which models the solidification and melting processes of certain metallic alloys that may solidify in two different kinds of crystallization; the model also takes in account the possibility of flow of the molten material due to thermal or concentration differences. This system is the following:

$$
\begin{array}{lr}
\tau_{t}-b \Delta \tau+v \cdot \nabla \tau=l_{1} \alpha_{t}+l_{2} \beta_{t}+l_{3} \gamma_{t}+f, & \text { in } Q, \\
\alpha_{t}-k \Delta \alpha+v \cdot \nabla \alpha=g_{1}(\tau, \alpha, \beta, \gamma)+g_{3}(\tau, \alpha, \beta, \gamma), & \text { in } Q, \\
\beta_{t}-k \Delta \beta+v \cdot \nabla \beta=g_{2}(\tau, \alpha, \beta, \gamma)-g_{3}(\tau, \alpha, \beta, \gamma), & \text { in } Q, \\
\gamma_{t}-k \Delta \gamma+v \cdot \nabla \gamma=-g_{1}(\tau, \alpha, \beta, \gamma)-g_{2}(\tau, \alpha, \beta, \gamma), & \text { in } Q, \\
v_{t}-\nu \Delta v+\nabla v+v \cdot \nabla v+h(\alpha+\beta) \cdot v=\mathcal{F}(\tau, \alpha, \beta, \gamma)+F, & \text { in } Q_{m l}, \\
\text { div } v=0, & \text { in } Q_{m l}, \\
v=0, & \text { in } Q_{s}, \\
\partial \tau / \partial n=\partial \alpha / \partial n=\partial \beta / \partial n=\partial \gamma / \partial n=0, & \text { on } \partial \Omega \times(0, T), \\
v=0, & \text { on } \partial Q_{m l}, \\
\tau=\tau_{0}, \alpha=\alpha_{0}, \beta=\beta_{0}, \gamma=\gamma_{0}, v=v_{0}, & \text { in } \Omega \times\{t=0\} .
\end{array}
$$

The notations used here are as follows. The set $\Omega \subset \mathbb{R}^{2}$ is a bounded $C^{2}$-domain,

${ }^{*}$ Received: March 23, 2012; accepted (in revised form); February 5, 2013. Communicated by Chun Liu.

${ }^{\dagger}$ FCA, University of Campinas, Pedro Zaccaria Street, 1300, Limeira - SP, CEP. 13484-350, Brazil (biancamrc@yahoo.com).

${ }^{\ddagger}$ IMECC, University of Campinas, Sergio Buarque de Holanda Street, 651, Cidade Universitária "Zeferino Vaz", Barão Geraldo, Campinas - SP, CEP. 13083-859, Brazil (boldrini@ime.unicamp.br). 
$0<T<\infty, Q=\Omega \times(0, T)$, and $n$ denotes the outer unitary normal to the boundary $\partial \Omega$.

The unknown function $\tau$ is associated to the temperature; the phase field unknown functions $\alpha$ and $\beta$ represent solid fractions of two different kinds of crystallization, while the also unknown phase field function $\gamma$ represents the liquid fraction; thus, by their physical meanings, we must have $\alpha, \beta, \gamma \geq 0$ and $\alpha+\beta+\gamma=1$. The velocity field is $v$.

The phase fields determine the solid and non solid regions: $Q_{s}=\{(x, t) \in Q$ : $\alpha(x, t)+\beta(x, t)=1\}$ gives the solid region, $Q_{m l}=\{(x, t) \in Q: \alpha(x, t)+\beta(x, t)<1\}$ gives the non-solid (molten) region. Note that the equations for the flow, which is assumed to be incompressible, is valid only in this unknown molten region $Q_{m l}$ while the velocity must be zero at the solid region $Q_{s}$.

In the equation for the temperature, equation (1.1) $(i), b>0$ is a given constant related to the thermal diffusion coefficient; $l_{1}, l_{2}$, and $l_{3}$ are given constants related to the latent heat of the associated to each kind of material states; the function $f$ is related to the given density of heat sources and sinks.

In the equations for the the phase fields, that is, equations (1.1) (ii), (iii), (iv), the given positive constant $k>0$ is related to the width of the transitions layers; interactions among the phase fields are given by the functions

$$
\begin{aligned}
& g_{1}=g_{1}(\tau, \alpha, \beta, \gamma)=-a_{1} \alpha \gamma\left(\gamma-\alpha+c_{1} \tau+d_{1}\right), \\
& g_{2}=g_{2}(\tau, \alpha, \beta, \gamma)=-a_{2} \beta \gamma\left(\gamma-\beta+c_{2} \tau+d_{2}\right), \\
& g_{3}=g_{3}(\tau, \alpha, \beta, \gamma)=-a_{3} \alpha \beta\left(\beta-\alpha+c_{3} \tau+d_{3}\right),
\end{aligned}
$$

where $a_{1}, a_{2}, a_{3}, c_{1}, c_{2}, c_{3}, d_{1}, d_{2}, d_{3}$ are given constants.

Observe that the equations for the temperature and phase fields present advection terms since we are considering the effects of the flow of the materials.

In the modified Navier-Stokes equations for the flow, equation $(1.1)(v)$, the viscosity $\nu>0$ is assumed to be constant; $F$ is a given external force field; to take in consideration the buoyancy forces due to thermal and concentrations differences, there is a Boussinesq type term:

$$
\mathcal{F}(\tau, \alpha, \beta, \gamma)=A \tau+B \alpha+C \beta+D \gamma,
$$

where $A, B, C$, and $D$ are given suitable functions. In this same equation, we also have a Carman-Kozeny type term $h(\alpha+\beta) v$ that acts as a penalization term and take care of the variation of the flow in the non-solid regions. This is done by introducing an internal dry friction that is null in pure liquid state and increases as the material approaches the solid state. Thus, the function $h(\cdot)$ is a given non-negative function such that $h(\alpha+\beta)$ must be zero at the pure liquid region $Q_{l}=\{(x, t) \in Q: \alpha(x, t)+\beta(x, t)=$ $0\}$, strictly positive in the mushy region $Q_{m l}=\{(x, t) \in Q: 0<\alpha(x, t)+\beta(x, t)<1\}$ and also $h(\alpha+\beta) \rightarrow+\infty$ as $\alpha+\beta \rightarrow 1^{-}$; that is $h(\cdot)$ is considered $\infty$ in the solid region $Q_{s}=\{(x, t) \in Q: \alpha(x, t)+\beta(x, t)=1\}$. Therefore, the Carman-Kozeny term brings a singularity to the equation, and this is the reason that it holds only in the a priori not known non solid region $Q_{m l}$. This term also forces the velocity to become zero on the solid region $Q_{s}$. Hence, the present problem is in fact a free-boundary value problem. We remark that the classical Carman-Kozeny term is $h(z)=C z^{2} /(1-z)^{3}$, where $C>0$ is a constant depending on the material.

The initial conditions $\tau_{0}, \alpha_{0}, \beta_{0}, \gamma_{0}$, and $v_{0}$ are suitable given functions that according to their physical interpretation satisfy $\alpha_{0}+\beta_{0}+\gamma_{0}=1$. 
The present model for alloy solidification involves more than one phase field and can be seen as a generalization of the model treated by (Hoffman and Jiang 1992) and of the one treated by Steinbach et al. in (Steinbach at al. 1996) and (Steinbach and Pezzolla 1999). As in (Hoffman and Jiang 1992), the present model assumes that the energy stored in the transition layers is isotropic, but in (Hoffman and Jiang 1992) only one crystallization type is allowed so that there is no possibility of flow in the melt. As in (Steinbach at al. 1996) and (Steinbach and Pezzolla 1999) we have two possible crystallization states and the same interactions potentials for the phase fields, and thus the same functions $g_{1}, g_{2}$, and $g_{3}$; but in (Steinbach at al. 1996) and (Steinbach and Pezzolla 1999) the temperature is supposed to be known and there is no possibility of flow in the melt. Besides, rigorous mathematical analysis is not the main interest in (Steinbach at al. 1996) and (Steinbach and Pezzolla 1999). We also call the attention of the reader to the fact that equations of (1.1) could be rewritten just in terms of $\alpha$ and $\beta$ since on physical grounds one must have $\gamma=1-\alpha-\beta$. Since in (Steinbach at al. 1996) and (Steinbach and Pezzolla 1999) it is presented using the three phase fields, for comparison we also preferred to present it as in (1.1).

To be fair, we must recall that there many articles considering phase field models. Here, we just mention some representative ones, dealing with different types and questions concerning phase field models: (Caginalp and Jones 1995), (Caginalp 1994), (Caginalp 1989), (Caginalp 1986), (Colli and Sprekel 1997), (Colli and Sprekel 1998), (Colli and Sprekel 1999), (Hoffman and Jiang 1992), (Horn et al. 1996), (Krejči et al. 2002), (Sprekel and Zheng 2003), (Planas and Boldrini 2005), (Planas 2007), (Boldrini and Planas 2002), (Boldrini and Planas 2005), (Boldrini and Vaz 2003), and (Vaz and Boldrini 2006). The interested reader can get more information by consulting the references of those papers.

Now, it is important to call the reader's attention to certain mathematical aspects of the problem.

The fact that it is a free-boundary problem for the flow due to the presence of a singularity term makes it impossible to get strong velocity estimates. Thus, only the usual estimate weak estimates for the velocity are available and, since the velocity appears in the advection terms appearing in the other equations, it also makes it harder to obtain stronger estimates for the other variables and thus to handle the nonlinearities. In fact, at a certain point of the arguments we will have to rely on local estimates for the time derivative of the velocity field. The fact that there is no strong estimates for the velocity also allows us to presently prove the existence of solutions for only the two-dimensional $(\mathrm{N}=2)$ case; the three-dimensional case is presently under investigation for a different constitutive law for the fluid flow; see (Caretta and Boldrini 2010).

From the point of view of the phase fields, the fact that here we have more than one of them brings another mathematical difficulty as compared to models with just one phase field. In fact, in this last case the higher power nonlinearities have the right sign at least for the process of obtaining the weaker estimates. On the other hand, here we also have higher powered nonlinearities which are products of different phase fields and thus we have no control of even their signs. These difficulties demand that we be very careful even to find the weaker estimates. We will also need to prove that the phase fields are nonnegative and add up to one.

We also remark that, unlike what occurs in the usual phase field models, in the present one there are terms in which the temperature acts by scalar multiplication on the phase fields, bringing nonlinearities that are harder to handle than the ones in 
the usual models.

To obtain existence of solutions for this model we combine ideas used in (Boldrini et al. 2009) to handle the fact that we have more than one phase field and in (Planas and Boldrini 2005) to handle the fact that we have flow in the melt. Our result is stated in Theorem 2.7 at the end of the next section.

This paper is organized as follows. In Section 2 we fix the notations, recall certain results that will be used along the paper, and state our main result concerning the existence of a suitable weak solution for the free-boundary value problem. In Section 3 we consider a sequence of regularized problems and obtain some estimates and convergence results that allow one to pass to a suitable limit. Finally, in Section 4 we pass to the limit in the previously obtained regularized solutions and get a weak solution of the original problem.

\section{Preliminaries, hypotheses, and main result}

For convenience of references, here we will just recall certain facts that will be useful along the paper.

We will use standard notations for Sobolev spaces. Given $1 \leq p \leq+\infty$ and $k \in$ $\mathbb{N}$, we denote the usual Sobolev space by $W_{p}^{k}(\Omega)=\left\{f \in L^{p}(\Omega): D^{\alpha} f \in L^{p}(\Omega),|\alpha| \leq k\right\}$, whose properties can be found for instance in (Adams 1975, Theorem 5.4, pp. 97); here we only mention the following result that is a consequence of the Sobolev embedding theorem given in (Adams 1975, Theorem 5.4, pp. 97).

Lemma 2.1. If $\Omega \subset \mathbb{R}^{2}$ satisfies the cone property and $p$ satisfies $1 \leq p<\infty$, then

$$
\begin{aligned}
& \text { (i) } W_{p}^{2}(\Omega) \hookrightarrow W_{q}^{2-\frac{2}{q}}(\Omega) \text {, for all } p \leq q \leq 2 p \text {; } \\
& \text { (ii) } W_{p}^{k}(\Omega) \hookrightarrow L^{\infty}(\Omega) \text {, for all } k, p>2 \text { with } k \in \mathbb{N} \text {; } \\
& \text { (iii) } H_{0}^{1}(\Omega) \hookrightarrow L^{q}(\Omega) \text {, for all } q \in \mathbb{N},
\end{aligned}
$$

with continuous embedding.

The first four equations in problem (1.1) will be studied in functional spaces denoted by $W_{q}^{2,1}(Q)=\left\{f \in L^{q}(Q): D^{\alpha} f \in L^{q}(Q), \forall 1 \leq|\alpha| \leq 2, f_{t} \in L^{q}(Q)\right\}$, and $L^{p}(0, T ; B)=\left\{f:(0, T) \rightarrow B:\|\| f(t)\left\|_{B}\right\|_{L^{p}((0, T))}<+\infty\right\}$, with $B$ any of several suitable Banach spaces.

Results concerning these spaces can be found for instance in (Ladyzhenskaya 1968) and (Mikhaylov 1978). Here we just recall a result that sometimes is called the Lions-Peetre embedding theorem (Lions 1985, pp. 15); it is also a consequence of Lemma 3.3, pp. 80, in (Ladyzhenskaya 1968).

Lemma 2.2. Let $\Omega \subset \mathbb{R}^{2}$ an open and bounded $C^{2}$-domain and let $Q=\Omega \times(0, T)$, with $0<T<\infty$. Then, $W_{q}^{2,1}(Q) \subset L^{p}(Q)$, with compact and continuous embedding for

$$
p=\left\{\begin{array}{cl}
\text { any positive number, } & \text { if } q=2, \\
\infty, & \text { if } q>2 .
\end{array}\right.
$$

To deal with the modified Navier-Stokes equations in problem (1.1), it is necessary to consider two other functional spaces. For each open set $U \subset \mathbb{R}^{2}$ let $\mathcal{V}(U)=\{w \in$ $\left(C^{\infty}(U)\right)^{2}$ : div $\left.w=0\right\}$. Then, $H(U)$ is the closure of $\mathcal{V}(U)$ in $\left(L^{2}(U)\right)^{2}$ and $V(U)$ is the closure of $\mathcal{V}(U)$ in $\left(H_{0}^{1}(U)\right)^{2}$. Besides,

$$
H:=H(\Omega), \quad V:=V(\Omega) .
$$


By using the Gagliardo-Nirenberg Inequality (see (Friedman 1976), pp. 27), we easily obtain the following result.

Lemma 2.3. Let $\Omega \subset \mathbb{R}^{2}$ an open and bounded $C^{1}$-domain. Then $L^{\infty}(0, T ; H) \cap$ $L^{2}(0, T ; V) \subset L^{4}(Q)$, with continuous imbedding, and satisfies

$$
\|v\|_{L^{4}(Q)} \leq C\|v\|_{L^{\infty}(0, T ; H)}^{1 / 2} \cdot\|v\|_{L^{2}(0, T ; V)}^{1 / 2},
$$

where $C$ depends only on $\Omega$.

Finally, let us state a result on existence, uniqueness, and regularity of solutions which is a special case of one for linear parabolic differential partial equations given by Theorem 9.1, pp. 341, in (Ladyzhenskaya 1968).

Proposition 2.4. Let $\Omega \subset \mathbb{R}^{2}$ an open and bounded domain. Suppose that $f \in$ $L^{q}(Q)$ and $c \in L^{q}(Q)$, with $q>1, \phi_{0} \in W_{q}^{2-2 / q}(\Omega)$ satisfies the compatibility condition $\partial \phi_{0} /\left.\partial n\right|_{\partial \Omega}=0$, and $v \in L^{r}(Q)$, with $r=\max \{q, 4\}$ if $q \neq 4$ and $r=4+\varepsilon$ for any $\varepsilon \geq 0$ if $q=4$. Then, the problem

$$
\left\{\begin{array}{lc}
\phi_{t}-k \Delta \phi+v \cdot \nabla \phi+c \phi=f, & \text { in } Q, \\
\partial \phi / \partial n=0, & \text { on } \partial \Omega \times(0, T), \\
\phi=\phi_{0}, & \text { in } \Omega \times\{t=0\} .
\end{array}\right.
$$

has a unique solution $\phi \in W_{q}^{2,1}(Q)$. This solution satisfies the estimate

$$
\|\phi\|_{W_{q}^{2,1}(Q)} \leq C\left[\|f\|_{L^{q}(Q)}+\left\|\phi_{0}\right\|_{W_{q}^{2-2 / q}(\Omega)}\right],
$$

where $C$ depends on $\Omega, T, k, v$, and $c$.

Next, we state a result on existence, uniqueness, and regularity of, and an a priori estimate for solutions of an auxiliary problem which is presented and proved in Calsavara and Boldrini [10]

Proposition 2.5. Assume that hypotheses (2.3) and (2.4) hold. Let $f \in L^{q}(Q)$, with $2<q<4$, and $v \in L^{4}(Q) \cap L^{2}(0, T ; V)$. Then there exists a unique solution $(\tau, \alpha, \beta, \gamma) \in$ $\left[W_{q}^{2,1}(Q)\right]^{4}$ of the problem

$$
\left\{\begin{array}{lc}
\tau_{t}-b \Delta \tau+v \cdot \nabla \tau=l_{1} \alpha_{t}+l_{2} \beta_{t}+l_{3} \gamma_{t}+f, & \text { in } Q, \\
\alpha_{t}-k \Delta \alpha+v \cdot \nabla \alpha=g_{1}(\tau, \alpha, \beta, \gamma)+g_{3}(\tau, \alpha, \beta, \gamma), & \text { in } Q, \\
\beta_{t}-k \Delta \beta+v \cdot \nabla \beta=g_{2}(\tau, \alpha, \beta, \gamma)-g_{3}(\tau, \alpha, \beta, \gamma), & \text { in } Q, \\
\gamma_{t}-k \Delta \gamma+v \cdot \nabla \gamma=-g_{1}(\tau, \alpha, \beta, \gamma)-g_{2}(\tau, \alpha, \beta, \gamma), & \text { in } Q, \\
\partial \tau / \partial n=\partial \alpha / \partial n=\partial \beta / \partial n=\partial \gamma / \partial n=0, & \text { on } \partial \Omega \times(0, T), \\
\tau=\tau_{0}, \alpha=\alpha_{0}, \beta=\beta_{0}, \gamma=\gamma_{0}, & \text { in } \Omega \times\{t=0\},
\end{array}\right.
$$

where $g_{1}, g_{2}$, and $g_{3}$ are given by (1.2).

Besides, this solution satisfies $\alpha \geq 0, \beta \geq 0, \gamma \geq 0, \alpha+\beta+\gamma=1$, and

$$
\begin{gathered}
\|\tau\|_{W_{q}^{2,1}(Q)}+\|\alpha\|_{W_{q}^{2,1}(Q)}+\|\beta\|_{W_{q}^{2,1}(Q)}+\|\gamma\|_{W_{q}^{2,1}(Q)} \\
\leq C\left(\left\|\tau_{0}\right\|_{W_{2}^{2}(\Omega)}+\left\|\alpha_{0}\right\|_{W_{2}^{2}(\Omega)}+\left\|\beta_{0}\right\|_{W_{2}^{2}(\Omega)}+\left\|\gamma_{0}\right\|_{W_{2}^{2}(\Omega)}+\|f\|_{L^{q}(Q)}\right),
\end{gathered}
$$


where the constant $C$ depends on $\Omega, T,\|v\|_{L^{4}(Q)}$, and the constants of Problem (2.1).

Remark 2.6. Under the hypothesis of Proposition 2.5, the solution $(\tau, \alpha, \beta, \gamma)$ of Problem (2.1) satisfies

$$
\begin{aligned}
& \int_{\Omega}\left(\tau^{2}(t)+\alpha^{2}(t)+\beta^{2}(t)+\gamma^{2}(t)\right) d x+\int_{0}^{t} \int_{\Omega}\left(|\nabla \tau|^{2}+|\nabla \alpha|^{2}+|\nabla \beta|^{2}+|\nabla \gamma|^{2}\right) d x d t \\
\leq & C\left(\left\|\tau_{0}\right\|_{L^{2}(\Omega)}^{2}+\left\|\alpha_{0}\right\|_{L^{2}(\Omega)}^{2}+\left\|\beta_{0}\right\|_{L^{2}(\Omega)}^{2}+\|f\|_{L^{2}(Q)}^{2}\right)+C \int_{0}^{t} \int_{\Omega}|v|^{2} d x d t,
\end{aligned}
$$

for all $0 \leq t \leq T$, where the constant $C$ depends on $\Omega, T$, and constants of problem (2.1).

Next, for easy reference, we collect in the following some hypotheses that will be assumed throughout the rest of this article.

\section{Main hypotheses:}

(i) $\Omega \subset \mathbb{R}^{2}$ is a bounded $C^{2}$-domain;

(ii) $0<T<\infty$, and $Q=\Omega \times(0, T)$;

(iii) $l_{1}$ (or $\left.l_{1}^{\prime}\right), l_{2}$ (or $\left.l_{2}^{\prime}\right), l_{3}, c_{1}, c_{2}, c_{3}, d_{1}, d_{2}$, and $d_{3}$ are real constants;

$b, k, a_{1}, a_{2}, a_{3}$, and $\nu$ are positive constants;

(iv) $h \in C^{1}([0,1))$ is a non-decreasing function such that $h(0)=0$ and $\lim _{\theta \rightarrow 1^{-}} h(\theta)=+\infty$;

(v) $A(\cdot), B(\cdot), C(\cdot)$, and $D(\cdot)$ are given functions in $\left(L^{\infty}(Q)\right)^{2}$;

(vi) $F(\cdot)$ is a given function in $\left(L^{2}(Q)\right)^{2}$.

For the initial conditions we assume that

$$
\begin{aligned}
& \text { (vii) } \tau_{0}, \alpha_{0}, \beta_{0}, \gamma_{0} \in W_{2}^{2}(\Omega) ; \\
& \text { (viii) } \alpha_{0}, \beta_{0}, \gamma_{0} \geq 0, \text { and } \alpha_{0}+\beta_{0}+\gamma_{0}=1 ; \\
& \text { (ix) } \partial \tau_{0} / \partial n=\partial \alpha_{0} / \partial n=\partial \beta_{0} / \partial n=\partial \gamma_{0} / \partial n=0 .
\end{aligned}
$$

For initial velocity field we require that

$$
\begin{aligned}
& \text { (x) } v_{0} \in H ; \\
& \text { (xi) } v_{0}(x)=0 \text { for all } x \in \Omega \text { such that } \alpha_{0}+\beta_{0}=1 .
\end{aligned}
$$

Let us observe that $\alpha_{0}+\beta_{0}=1$ implies $\gamma_{0}=0$, and the last condition means that the initial velocity is null in the initial solid region.

Our objective in this work is to show the following existence result for weak solutions for Problem (1.1) under the previous hypotheses.

TheOREM 2.7. Under hypotheses (2.3), (2.4), (2.5), and $f \in L^{q}(Q)$, with $2<q<4$, there are functions $\tau, \alpha, \beta, \gamma \in W_{q}^{2,1}(Q)$, with $\alpha \geq 0, \beta \geq 0, \gamma \geq 0, \alpha+\beta+\gamma=1$ in $Q$, and $v \in L^{\infty}(0, T ; H) \cap L^{2}(0, T ; V)$ such that $(v, \tau, \alpha, \beta, \gamma)$ is a solution of problem (1.1) in the following weak sense:

If 


$$
\begin{aligned}
& Q_{m l}=\{(x, t) \in Q: \alpha(x, t)+\beta(x, t)<1\}, \\
& Q_{s}=\{(x, t) \in Q: \alpha(x, t)+\beta(x, t)=1\}, \\
& \Omega_{m l}(0)=\left\{(x, 0): x \in \Omega, \alpha_{0}(x)+\beta_{0}(x)<1\right\} \text {, } \\
& \text { then } \\
& \tau_{t}-b \Delta \tau+v \cdot \nabla \tau=l_{1} \alpha_{t}+l_{2} \beta_{t}+l_{3} \gamma_{t}+f, \\
& \text { in } L^{2}(Q) \text {, } \\
& \alpha_{t}-k \Delta \alpha+v \cdot \nabla \alpha=g_{1}(\tau, \alpha, \beta, \gamma)+g_{3}(\tau, \alpha, \beta, \gamma), \\
& \text { in } L^{2}(Q) \text {, } \\
& \beta_{t}-k \Delta \beta+v \cdot \nabla \beta=g_{2}(\tau, \alpha, \beta, \gamma)-g_{3}(\tau, \alpha, \beta, \gamma), \\
& \text { in } L^{2}(Q) \text {, } \\
& \gamma_{t}-k \Delta \gamma+v \cdot \nabla \gamma=-g_{1}(\tau, \alpha, \beta, \gamma)-g_{2}(\tau, \alpha, \beta, \gamma), \\
& \text { in } L^{2}(Q) \text {, } \\
& \partial \tau / \partial n=\partial \alpha / \partial n=\partial \beta / \partial n=\partial \gamma / \partial n=0, \quad \text { on } \partial \Omega \times(0, T) \text {, } \\
& \tau=\tau_{0}, \alpha=\alpha_{0}, \beta=\beta_{0}, \gamma=\gamma_{0}, \quad \text { in } \Omega \times\{t=0\} . \\
& -\int_{0}^{T}<v, \eta_{t}>d s+\nu \int_{0}^{T}(\nabla v, \nabla \eta) d s+\int_{0}^{T}(v \cdot \nabla v, \eta) d s+\int_{0}^{T}(h(\alpha+\beta) . v, \eta) d s \\
& =\int_{0}^{T}(\mathcal{F}(\tau, \alpha, \beta, \gamma), \eta) d s+\int_{0}^{T}(F, \eta) d s \quad+\left(v_{0}, \eta(0)\right)_{\Omega_{m l}(0)}, \quad \forall \eta \in \mathcal{W}_{m l}, \\
& v=0, \\
& \text { in } Q_{s} \text {. }
\end{aligned}
$$

Here,

$$
\begin{aligned}
\mathcal{W}_{m l}= & \left\{\eta \in L^{2}(0, T ; V): \eta_{t} \in L^{2}\left(0, T, V^{\prime}\right),\right. \text { and } \\
& \left.\eta(T)=0 \text { with compact supp } \eta \subset Q_{m l} \cup \Omega_{m l}(0)\right\},
\end{aligned}
$$

and $g_{1}, g_{2}$, and $g_{3}$ are given by (1.2).

\section{A regularized problem}

In this section we introduce a regularized version of the original problem by modifying the Navier-Stokes type equations in such way that they hold in the whole domain $\Omega$ instead of only in an a priori unknown region.

For each $\delta \in(0, \varepsilon)$, with $0<\varepsilon \leq 1$, let us consider the following regularized problem:

$$
\begin{aligned}
& \tau_{t}^{\delta}-b \Delta \tau^{\delta}+v^{\delta} \cdot \nabla \tau^{\delta}=l_{1} \alpha_{t}^{\delta}+l_{2} \beta_{t}^{\delta}+l_{3} \gamma_{t}^{\delta}+f, \quad \text { in } Q, \\
& \alpha_{t}^{\delta}-k \Delta \alpha^{\delta}+v^{\delta} \cdot \nabla \alpha^{\delta}=g_{1}\left(\tau^{\delta}, \alpha^{\delta}, \beta^{\delta}, \gamma^{\delta}\right)+g_{3}\left(\tau^{\delta}, \alpha^{\delta}, \beta^{\delta}, \gamma^{\delta}\right), \quad \text { in } Q, \\
& \beta_{t}^{\delta}-k \Delta \beta^{\delta}+v^{\delta} \cdot \nabla \beta^{\delta}=g_{2}\left(\tau^{\delta}, \alpha^{\delta}, \beta^{\delta}, \gamma^{\delta}\right)-g_{3}\left(\tau^{\delta}, \alpha^{\delta}, \beta^{\delta}, \gamma^{\delta}\right), \quad \text { in } Q, \\
& \gamma_{t}^{\delta}-k \Delta \gamma^{\delta}+v^{\delta} \cdot \nabla \gamma^{\delta}=-g_{1}\left(\tau^{\delta}, \alpha^{\delta}, \beta^{\delta}, \gamma^{\delta}\right)-g_{2}\left(\tau^{\delta}, \alpha^{\delta}, \beta^{\delta}, \gamma^{\delta}\right) \text {, in } Q \text {, } \\
& \partial \tau^{\delta} / \partial n=\partial \alpha^{\delta} / \partial n=\partial \beta^{\delta} / \partial n=\partial \gamma^{\delta} / \partial n=0, \quad \text { on } \partial \Omega \times(0, T) \text {, } \\
& \tau^{\delta}=\tau_{0}, \alpha^{\delta}=\alpha_{0}, \beta^{\delta}=\beta_{0}, \gamma^{\delta}=\gamma_{0}, \quad \text { in } \Omega \times\{t=0\}, \\
& \frac{d}{d t}\left(v^{\delta}, u\right)+\nu\left(\nabla v^{\delta}, \nabla u\right)+\left(v^{\delta} . \nabla v^{\delta}, u\right)+\left(h\left(\alpha^{\delta}+\beta^{\delta}-\delta\right) v^{\delta}, u\right) \\
& =\left(\mathcal{F}\left(\tau^{\delta}, \alpha^{\delta}, \beta^{\delta}, \gamma^{\delta}\right), u\right)+(F, u), \quad \forall u \in V, \forall t \in(0, T), \\
& v^{\delta}=0, \quad \text { on } \partial \Omega \times(0, T), \\
& v^{\delta}=v_{0}, \quad \text { in } \Omega \times\{t=0\} .
\end{aligned}
$$


Here $g_{1}, g_{2}$, and $g_{3}$ are given by $(1.2), \mathcal{F}$ is given by (1.3), and $h$ is extended to be zero in $(-\varepsilon, 0)$, so $h(t) \geq 0$ for $t \in(-\varepsilon, 1)$.

The idea for this regularization comes from the fact that by item (iv) in Hypotheses $(2.3)$ we have that $h \in C^{1}([0,1))$ is a non-decreasing function such that $h(0)=0$ and $\lim _{x \rightarrow 1^{-}} h(x)=+\infty$. Since hopefully we will get $0 \leq \alpha^{\delta}+\beta^{\delta} \leq 1$, we will have that $h\left(\alpha^{\delta}+\beta^{\delta}-\delta\right)$ is bounded for each $\delta \in(0, \varepsilon)$.

For this regularized problem, the following result holds.

Proposition 3.1. Assume that hypotheses (2.3), (2.4), and (2.5) hold, and $f \in$ $L^{q}(Q)$, with $2<q<4$. For each $\delta \in(0, \varepsilon)$, the regularized problem (3.1) has a unique solution $\left(v^{\delta}, \tau^{\delta}, \alpha^{\delta}, \beta^{\delta}, \gamma^{\delta}\right)$ such that $v^{\delta} \in L^{\infty}(0, T ; H) \cap L^{2}(0, T ; V), v_{t}^{\delta} \in L^{2}\left(0, T ; V^{\prime}\right)$, $\left(\tau^{\delta}, \alpha^{\delta}, \beta^{\delta}, \gamma^{\delta}\right) \in\left[W_{q}^{2,1}(Q)\right]^{4}$,

$$
\alpha^{\delta} \geq 0, \beta^{\delta} \geq 0, \gamma^{\delta} \geq 0, \quad \text { and } \quad \alpha^{\delta}+\beta^{\delta}+\gamma^{\delta}=1 \quad \text { a.e. in } \quad Q .
$$

Besides, it holds that

$$
\begin{gathered}
\left\|v^{\delta}\right\|_{L^{\infty}(0, T ; H)}+\left\|v^{\delta}\right\|_{L^{2}(0, T ; V)} \\
+\left\|\tau^{\delta}\right\|_{W_{q}^{2,1}(Q)}+\left\|\alpha^{\delta}\right\|_{W_{q}^{2,1}(Q)}+\left\|\beta^{\delta}\right\|_{W_{q}^{2,1}(Q)}+\left\|\gamma^{\delta}\right\|_{W_{q}^{2,1}(Q)} \\
\leq C\left[\left\|v_{0}\right\|_{L^{2}(\Omega)}+\left\|\tau_{0}\right\|_{W_{2}^{2}(\Omega)}+\left\|\alpha_{0}\right\|_{W_{2}^{2}(\Omega)}+\left\|\beta_{0}\right\|_{W_{2}^{2}(\Omega)}+\left\|\gamma_{0}\right\|_{W_{2}^{2}(\Omega)}\right. \\
\left.+\|f\|_{L^{q}(Q)}+\|F\|_{L^{2}(Q)}\right]
\end{gathered}
$$

where $C$ depends on $T, \Omega,\left\|v_{0}\right\|_{L^{2}(\Omega)},\left\|\tau_{0}\right\|_{L^{2}(\Omega)},\left\|\alpha_{0}\right\|_{L^{2}(\Omega)},\left\|\beta_{0}\right\|_{L^{2}(\Omega)},\left\|\gamma_{0}\right\|_{L^{2}(\Omega)}$, $\|f\|_{L^{2}(Q)}$, and $\|F\|_{L^{2}(Q)}$, and constants of Problem (3.1), and thus is independent of $\delta$.

In the following arguments, we fix $\delta \in(0, \varepsilon)$ and for simplicity of notations in the rest of this section we will omit the superscript $\delta$ of $v^{\delta}, \tau^{\delta}, \alpha^{\delta}, \beta^{\delta}$, and $\gamma^{\delta}$.

To prove the last proposition, i.e, to obtain the existence of solutions of problem (3.1), let us apply the Leray-Schauder's fixed point theorem (Friedman 1964) in the following setting.

Consider the set

$$
\mathcal{K}:=L^{2}(0, T ; H) \times L^{\infty}(Q) \times \mathbb{K}
$$

where $\mathbb{K}=\left\{(\alpha, \beta, \gamma) \in\left[L^{\infty}(Q)\right]^{3}: \alpha, \beta, \gamma \geq 0\right.$ and $\left.\alpha+\beta+\gamma=1\right\}$, which is a closed convex subset of $L^{2}(0, T ; H) \times\left[L^{\infty}(Q)\right]^{4}$.

We also consider the family of operators $\mathcal{S}_{\lambda}: \mathcal{K} \rightarrow \mathcal{K}$, for $0 \leq \lambda \leq 1$, defined for any $(\widetilde{v}, \widetilde{\tau}, \widetilde{\alpha}, \widetilde{\beta}, \widetilde{\gamma}) \in \mathcal{K}$ as

$$
\mathcal{S}_{\lambda}(\widetilde{v}, \widetilde{\tau}, \widetilde{\alpha}, \widetilde{\beta}, \widetilde{\gamma})=(v, \tau, \alpha, \beta, \gamma),
$$

where $(v, \tau, \alpha, \beta, \gamma)$ is the solution of the following problem:

$$
\begin{aligned}
& \tau_{t}-b \Delta \tau+v \cdot \nabla \tau=l_{1} \alpha_{t}+l_{2} \beta_{t}+l_{3} \gamma_{t}+f, \text { in } Q, \\
& \alpha_{t}-k \Delta \alpha+v \cdot \nabla \alpha=g_{1}(\tau, \alpha, \beta, \gamma)+g_{3}(\tau, \alpha, \beta, \gamma), \quad \text { in } Q, \\
& \beta_{t}-k \Delta \beta+v \cdot \nabla \beta=g_{2}(\tau, \alpha, \beta, \gamma)-g_{3}(\tau, \alpha, \beta, \gamma), \quad \text { in } Q, \\
& \gamma_{t}-k \Delta \gamma+v \cdot \nabla \gamma=-g_{1}(\tau, \alpha, \beta, \gamma)-g_{2}(\tau, \alpha, \beta, \gamma), \quad \text { in } Q,
\end{aligned}
$$




$$
\begin{gathered}
\frac{d}{d t}(v, u)-\nu(\nabla v, \nabla u) \quad+(v \cdot \nabla v, u)+\lambda(h(\widetilde{\alpha}+\widetilde{\beta}-\delta) v, u) \\
=\lambda(\mathcal{F}(\widetilde{\tau}, \widetilde{\alpha}, \widetilde{\beta}, \widetilde{\gamma})+F, u), \quad \text { for all } u \in V, t \in(0, T), \\
\partial \tau / \partial n=\partial \alpha / \partial n=\partial \beta / \partial n=\partial \gamma / \partial n=0, \quad \text { on } \partial \Omega \times(0, T), \\
\tau=\tau_{0}, \alpha=\alpha_{0}, \quad \beta=\beta_{0} \quad \text { in } \Omega \times\{t=0\},
\end{gathered}
$$

where $g_{1}, g_{2}, g_{3}$, and $\mathcal{F}$ are given by (1.2) and (1.3).

In the next sequence of lemmas, we will verify the hypotheses of the LeraySchauder theorem.

LEMma 3.2. Assume that hypotheses (2.3), (2.4), and (2.5) hold. Let $f \in L^{q}(Q)$, with $2<q<4$. Then $\mathcal{S}_{\lambda}(\widetilde{v}, \widetilde{\tau}, \widetilde{\alpha}, \widetilde{\beta}, \widetilde{\gamma})$ is well defined for each $(\widetilde{v}, \widetilde{\tau}, \widetilde{\alpha}, \widetilde{\beta}, \widetilde{\gamma}) \in \mathcal{K}$ and each $0 \leq \lambda \leq 1$.

Proof. Indeed, since $\mathcal{F}(\widetilde{\tau}, \widetilde{\alpha}, \widetilde{\beta}, \widetilde{\gamma})+F \in L^{2}(Q)$, we have from theorems 3.1 and 3.2 in (Temam 1979), pp. 282 and 294, that there exists a unique $v \in L^{\infty}(0, T ; H) \cap L^{2}(0, T ; V)$ such that $v_{t} \in L^{2}\left(0, T ; V^{\prime}\right)$ is a solution of the fourth equation of problem (3.3). In particular, $v \in L^{2}(0, T ; H)$.

Now, since $v \in L^{\infty}(0, T ; H) \cap L^{2}(0, T ; V)$ by Lemma 2.3 , we have that $v \in L^{4}(Q)$. Then, by Proposition 2.5 applied to first, second, and third equations of problem (3.3), there exists a unique solution $(\tau, \alpha, \beta) \in\left[W_{q}^{2,1}(Q)\right]^{3}$ of these equations and this solution satisfies $\alpha, \beta, \gamma \geq 0$, and $\alpha+\beta+\gamma=1$. Besides, since $q>2$, we have $(\tau, \alpha, \beta, \gamma) \in$ $\left[L^{\infty}(Q)\right]^{4}$.

So, $(v, \tau, \alpha, \beta, \gamma) \in \mathcal{K}$ and it is the unique solution of Problem (3.3).

LEMmA 3.3. Assume that hypotheses (2.3), (2.4), and (2.5) hold. Let $f \in L^{q}(Q)$, with $2<q<4$. Then for each fixed $\lambda \in[0,1], \mathcal{S}_{\lambda}: \mathcal{K} \rightarrow \mathcal{K}$ is continuous and compact.

Proof. For this, we fix $\lambda \in[0,1]$, consider $\left(\widetilde{v}_{1}, \widetilde{\tau}_{1}, \widetilde{\alpha}_{1}, \widetilde{\beta}_{1}\right),\left(\widetilde{v}_{2}, \widetilde{\tau}_{2}, \widetilde{\alpha}_{2}, \widetilde{\beta}_{2}\right) \in \mathcal{K}$, with $\left(v_{i}, \tau_{i}, \alpha_{i}, \beta_{i}\right)=\mathcal{S}_{\lambda}\left(\widetilde{v}_{i}, \widetilde{\tau}_{i}, \widetilde{\alpha}_{i}, \widetilde{\beta}_{i}\right)$, for $i=1,2$, and define

$$
\begin{aligned}
& (\widetilde{v}, \widetilde{\tau}, \widetilde{\alpha}, \widetilde{\beta})=\left(\widetilde{v}_{1}, \widetilde{\tau}_{1}, \widetilde{\alpha}_{1}, \widetilde{\beta}_{1}\right)-\left(\widetilde{v}_{2}, \widetilde{\tau}_{2}, \widetilde{\alpha}_{2}, \widetilde{\beta}_{2}\right), \\
& (v, \tau, \alpha, \beta)=\left(v_{1}, \tau_{1}, \alpha_{1}, \beta_{1}\right)-\left(v_{2}, \tau_{2}, \alpha_{2}, \beta_{2}\right) .
\end{aligned}
$$

We have that $v \in L^{\infty}(0, T ; H) \cap L^{2}(0, T ; V)$ and satisfies

$$
\begin{aligned}
& \frac{d}{d t}(v, u)-\nu(\nabla v, \nabla u)+\left(v \cdot \nabla v_{1}, u\right)+\left(v_{2} \cdot \nabla v, u\right)+\lambda\left(h\left(\widetilde{\alpha}_{1}+\widetilde{\beta}_{1}-\delta\right) v, u\right) \\
= & -\lambda\left(\left(h\left(\widetilde{\alpha}_{1}+\widetilde{\beta}_{1}-\delta\right)-h\left(\widetilde{\alpha}_{2}+\widetilde{\beta}_{2}-\delta\right)\right) v_{2}, u\right)+\lambda(\mathcal{F}(\widetilde{\tau}, \widetilde{\alpha}, \widetilde{\beta}), u)
\end{aligned}
$$

for all $u \in V, t \in(0, T)$.

By taking $u=v(t)$, for each $t \in(0, T)$ in the last equation, we obtain that

$$
\begin{gathered}
\frac{1}{2} \frac{d}{d t}\|v\|_{L^{2}(\Omega)}^{2}-\nu\|\nabla v\|_{L^{2}(\Omega)}^{2}+\left(v_{2} \cdot \nabla v, v\right)+\lambda\left(h\left(\widetilde{\alpha}_{1}+\widetilde{\beta}_{1}-\delta\right) v, v\right) \\
=-\left(v \cdot \nabla v_{1}, v\right)-\lambda\left(\left(h\left(\widetilde{\alpha}_{1}+\widetilde{\beta}_{1}-\delta\right)-h\left(\widetilde{\alpha}_{2}+\widetilde{\beta}_{2}-\delta\right)\right) v_{2}, v\right)+\lambda(\mathcal{F}(\widetilde{\tau}, \widetilde{\alpha}, \widetilde{\beta}), v) .
\end{gathered}
$$


Now, we observe that

$$
\begin{aligned}
& \left(v_{2} \cdot \nabla v, v\right)=0, \\
& \left(h\left(\widetilde{\alpha}_{1}+\widetilde{\beta}_{1}-\delta\right) v, v\right) \geq 0, \\
& \left|\left(v \cdot \nabla v_{1}, v\right)\right| \leq\|v\|_{L^{4}(\Omega)}^{2}\left\|\nabla v_{1}\right\|_{L^{2}(\Omega)} \leq\|v\|_{L^{2}(\Omega)}\|\nabla v\|_{L^{2}(\Omega)}\left\|\nabla v_{1}\right\|_{L^{2}(\Omega)} \\
& \leq C_{\varepsilon}\|v\|_{L^{2}(\Omega)}^{2}\left\|\nabla v_{1}\right\|_{L^{2}(\Omega)}^{2}+\varepsilon\|\nabla v\|_{L^{2}(\Omega)}^{2} \\
& \leq C_{\varepsilon}\|v\|_{L^{2}(\Omega)}^{2}+\varepsilon\|\nabla v\|_{L^{2}(\Omega)}^{2}, \\
& \left(\left(h\left(\widetilde{\alpha}_{1}+\widetilde{\beta}_{1}-\delta\right)-h\left(\widetilde{\alpha}_{2}+\widetilde{\beta}_{2}-\delta\right)\right) v_{2}, v\right) \\
& =\left(h^{\prime}(\theta)(\widetilde{\alpha}+\widetilde{\beta}-\delta) v_{2}, v\right) \\
& \leq C\left(\|\widetilde{\alpha}\|_{L^{2}(\Omega)}+\|\widetilde{\beta}\|_{L^{2}(\Omega)}\right)\left\|v_{2}\right\|_{L^{4}(\Omega)}\|v\|_{L^{4}(\Omega)} \\
& \leq C\left(\|\widetilde{\alpha}\|_{L^{2}(\Omega)}+\|\widetilde{\beta}\|_{L^{2}(\Omega)}\right)\left\|\nabla v_{2}\right\|_{L^{2}(\Omega)}\|\nabla v\|_{L^{2}(\Omega)} \\
& \leq C_{\varepsilon}\|\widetilde{\alpha}\|_{L^{2}(\Omega)}^{2}+C_{\varepsilon}\|\widetilde{\beta}\|_{L^{2}(\Omega)}^{2}+\varepsilon\left\|\nabla v_{2}\right\|_{L^{2}(\Omega)}^{2}\|\nabla v\|_{L^{2}(\Omega)}^{2} \\
& \leq C_{\varepsilon}\|\widetilde{\alpha}\|_{L^{2}(\Omega)}^{2}+C_{\varepsilon}\|\widetilde{\beta}\|_{L^{2}(\Omega)}^{2}+C \cdot \varepsilon\|\nabla v\|_{L^{2}(\Omega)}^{2}, \\
& (\mathcal{F}(\widetilde{\tau}, \widetilde{\alpha}, \widetilde{\beta}), v) \leq C\left(\|\widetilde{\tau}\|_{L^{2}(\Omega)}^{2}+\|\widetilde{\alpha}\|_{L^{2}(\Omega)}^{2}+\|\widetilde{\beta}\|_{L^{2}(\Omega)}^{2}+\|v\|_{L^{2}(\Omega)}^{2}\right),
\end{aligned}
$$

where $\theta \in[0,1]$.

By replacing the last equalities and inequalities in the previous one, we obtain

$$
\begin{aligned}
\frac{d}{d t}\|v\|_{L^{2}(\Omega)}^{2}+\nu\|\nabla v\|_{L^{2}(\Omega)}^{2} \leq & C_{\epsilon}\|v\|_{L^{2}(\Omega)}^{2}+C . \epsilon\|\nabla \widetilde{v}\|_{L^{2}(\Omega)}^{2} \\
& +C\|\widetilde{\tau}\|_{L^{2}(\Omega)}^{2}+C_{\epsilon}\|\widetilde{\alpha}\|_{L^{2}(\Omega)}^{2}+C_{\epsilon}\|\widetilde{\beta}\|_{L^{2}(\Omega)}^{2} .
\end{aligned}
$$

By taking $\epsilon>0$ small enough and integrating in $(0, t)$, with $0 \leq t \leq T$, it follows that

$$
\begin{aligned}
\|v(t)\|_{L^{2}(\Omega)}^{2}+\|\nabla v(t)\|_{L^{2}(\Omega)}^{2} \leq & C\left(\|\widetilde{\alpha}\|_{L^{2}(Q)}^{2}+\|\widetilde{\beta}\|_{L^{2}(Q)}^{2}+\|\widetilde{\tau}\|_{L^{2}(Q)}^{2}\right) \\
& +C \int_{0}^{t}\|v\|_{L^{2}(\Omega)}^{2} d t .
\end{aligned}
$$

By Gronwall's lemma, we get for all $t \in(0, T)$ that

$$
\|v(t)\|_{L^{2}(\Omega)}^{2}+\|\nabla v(t)\|_{L^{2}(\Omega)}^{2} \leq C\left(\|\widetilde{\alpha}\|_{L^{2}(Q)}^{2}+\|\widetilde{\beta}\|_{L^{2}(Q)}^{2}+\|\widetilde{\tau}\|_{L^{2}(Q)}^{2}\right) .
$$

Thus,

$$
\|v\|_{L^{2}(0, T ; H)} \leq C\left(\|v\|_{L^{\infty}(0, T ; H)}+\|v\|_{\left.L^{2}(0, T ; V)\right)}\right) \leq C\|(\widetilde{v}, \widetilde{\tau}, \widetilde{\alpha}, \widetilde{\beta})\|_{\mathcal{K}}
$$

and

$$
\|v\|_{L^{4}(Q)} \leq C\|v\|_{L^{\infty}(0, T ; H)}^{1 / 2}\|v\|_{\left.L^{2}(0, T ; V)\right)}^{1 / 2} \leq C\|(\widetilde{v}, \widetilde{\tau}, \widetilde{\alpha}, \widetilde{\beta})\|_{\mathcal{K}}
$$


Next, we observe that from the fact that $\alpha$ satisfies

$$
\begin{gathered}
\alpha_{t}-k \Delta \alpha+v \cdot \nabla \alpha \\
=-a_{1}\left[\alpha_{1} \gamma_{1}\left(\gamma_{1}-\alpha_{1}+c_{1} \tau_{1}+d_{1}\right)-\alpha_{2} \gamma_{2}\left(\gamma_{2}-\alpha_{2}+c_{1} \tau_{2}+d_{1}\right)\right] \\
-a_{3}\left[\alpha_{1} \beta_{1}\left(\beta_{1}-\alpha_{1}+c_{3} \tau_{1}+d_{3}\right)-\alpha_{2} \beta_{2}\left(\beta_{2}-\alpha_{2}+c_{3} \tau_{2}+d_{3}\right)\right], \quad \text { in } Q, \\
\partial \alpha / \partial n=0, \quad \text { on } \partial \Omega \times(0, T), \\
\alpha=0, \quad \text { in } \Omega \times\{t=0\},
\end{gathered}
$$

where $a_{1}, a_{2}, a_{3}, c_{1}, c_{2}, c_{3}, d_{1}, d_{2}$, and $d_{3}$ are given constants, by adding and subtracting suitable terms, we obtain that $\alpha$ satisfies

$$
\begin{aligned}
& \alpha_{t}-k \Delta \alpha+v_{2} \cdot \nabla \alpha \\
& \quad+\left[a_{1} \gamma_{1}\left(\gamma_{1}-\alpha_{1}+c_{1} \tau_{1}+d_{1}\right)-a_{1} \alpha_{2} \gamma_{2}+a_{3} \beta_{1}\left(\beta_{1}-\alpha_{1}+c_{3} \tau_{1}+d_{3}\right)-a_{3} \alpha_{2} \beta_{2}\right] \alpha \\
& =-v \cdot \nabla \alpha_{1}+\beta\left[-a_{3} \alpha_{2}\left(\beta_{1}-\alpha_{1}+c_{3} \tau_{1}+d_{3}\right)-a_{3} \alpha_{2} \beta_{2}\right] \\
& +\gamma\left[-a_{1} \alpha_{2}\left(\gamma_{1}-\alpha_{1}+c_{1} \tau_{1}+d_{1}\right)-a_{1} \alpha_{2} \gamma_{2}\right]+\tau\left[-a_{1} c_{1} \alpha_{2} \gamma_{2}-a_{3} c_{3} \alpha_{2} \beta_{2}\right], \quad \text { in } Q, \\
& \partial \alpha / \partial n=0, \quad \text { on } \partial \Omega \times(0, T), \\
& \alpha=0, \quad \text { in } \Omega \times\{t=0\} .
\end{aligned}
$$

By using Proposition 2.4 with this last equation, estimate (2.2), and the continuous imbedding $W_{q}^{2,1}(Q) \subset L^{\infty}(Q)$, we conclude that for all $2<q<4$ it holds that

$$
\|\alpha\|_{W_{q}^{2,1}(Q)} \leq C\|\tau\|_{L^{q}(Q)}+C\|\beta\|_{L^{q}(Q)}+C\|\gamma\|_{L^{q}(Q)}+C\left\|v \cdot \nabla \alpha_{2}\right\|_{L^{q}(Q)},
$$

where $C$ depends on $\left\|\left(\widetilde{v}_{1}, \widetilde{\tau}_{1}, \widetilde{\alpha}_{1}, \widetilde{\beta}_{1}, \widetilde{\gamma}_{1}\right)\right\|_{\mathcal{K}}$ and $\left\|\left(\widetilde{v}_{2}, \widetilde{\tau}_{2}, \widetilde{\alpha}_{2}, \widetilde{\beta}_{2}, \widetilde{\gamma}_{2}\right)\right\|_{\mathcal{K}}$.

Next, recall that since $\Omega \in \mathbb{R}^{2}$, then $H_{0}^{1}(\Omega) \subset L^{r}(\Omega)$, for all $1 \leq r<\infty$; by fixing $q \in(2,4)$ and using Hölder's inequality first in $\Omega$ and then in $(0, T)$ for last term of the last equation, and using Gagliardo-Nirenberg's equations for the other three terms, we obtain

$$
\begin{gathered}
\|\alpha\|_{W_{q}^{2,1}(Q)} \leq C_{\varepsilon}\|\tau\|_{L^{2}(Q)}+\varepsilon\|\tau\|_{W_{q}^{2,1}(Q)}+C_{\varepsilon}\|\beta\|_{L^{2}(Q)}+\varepsilon\|\beta\|_{W_{q}^{2,1}(Q)} \\
+C_{\varepsilon}\|\gamma\|_{L^{2}(Q)}+\varepsilon\|\gamma\|_{W_{q}^{2,1}(Q)}+C\|v\|_{L^{4}(Q)}\left\|\alpha_{2}\right\|_{W_{2}^{2,1}(Q)} \\
\leq C_{\varepsilon}\|\tau\|_{L^{2}(Q)}+\varepsilon\|\tau\|_{W_{q}^{2,1}(Q)}+C_{\varepsilon}\|\beta\|_{L^{2}(Q)}+\varepsilon\|\beta\|_{W_{q}^{2,1}(Q)} \\
+C_{\varepsilon}\|\gamma\|_{L^{2}(Q)}+\varepsilon\|\gamma\|_{W_{q}^{2,1}(Q)}+C\|(\widetilde{v}, \widetilde{\tau}, \widetilde{\alpha}, \widetilde{\beta}, \widetilde{\gamma})\|_{\mathcal{K}},
\end{gathered}
$$

where $C$ depends on $\left\|\left(\widetilde{v}_{1}, \widetilde{\tau}_{1}, \widetilde{\alpha}_{1}, \widetilde{\beta}_{1}, \widetilde{\gamma}_{1}\right)\right\|_{\mathcal{K}}$ and $\left\|\left(\widetilde{v}_{2}, \widetilde{\tau}_{2}, \widetilde{\alpha}_{2}, \widetilde{\beta}_{2}, \widetilde{\gamma}_{2}\right)\right\|_{\mathcal{K}}$, by estimate $(3.5)$.

By proceeding similarly for equations satisfied by $\beta, \gamma$, and $\tau$, we also obtain

$$
\begin{array}{r}
\|\beta\|_{W_{q}^{2,1}(Q)} \leq C_{\varepsilon}\|\tau\|_{L^{2}(Q)}+\varepsilon\|\tau\|_{W_{q}^{2,1}(Q)}+C_{\varepsilon}\|\alpha\|_{L^{2}(Q)}+\varepsilon\|\alpha\|_{W_{q}^{2,1}(Q)} \\
+C_{\varepsilon}\|\gamma\|_{L^{2}(Q)}+\varepsilon\|\gamma\|_{W_{q}^{2,1}(Q)}+C\|(\widetilde{v}, \widetilde{\tau}, \widetilde{\alpha}, \widetilde{\beta}, \widetilde{\gamma})\|_{\mathcal{K}}, \\
\|\gamma\|_{W_{q}^{2,1}(Q)} \leq C_{\varepsilon}\|\tau\|_{L^{2}(Q)}+\varepsilon\|\tau\|_{W_{q}^{2,1}(Q)}+C_{\varepsilon}\|\alpha\|_{L^{2}(Q)}+\varepsilon\|\alpha\|_{W_{q}^{2,1}(Q)} \\
+C_{\varepsilon}\|\beta\|_{L^{2}(Q)}+\varepsilon\|\beta\|_{W_{q}^{2,1}(Q)}+C\|(\widetilde{v}, \widetilde{\tau}, \widetilde{\alpha}, \widetilde{\beta}, \widetilde{\gamma})\|_{\mathcal{K}},
\end{array}
$$


and

$$
\begin{array}{r}
\|\tau\|_{W_{q}^{2,1}(Q)} \leq C_{\varepsilon}\|\alpha\|_{L^{2}(Q)}+\varepsilon\|\alpha\|_{W_{q}^{2,1}(Q)}+C_{\varepsilon}\|\beta\|_{L^{2}(Q)}+\varepsilon\|\beta\|_{W_{q}^{2,1}(Q)} \\
+C_{\varepsilon}\|\gamma\|_{L^{2}(Q)}+\varepsilon\|\gamma\|_{W_{q}^{2,1}(Q)}+C\|(\widetilde{v}, \widetilde{\tau}, \widetilde{\alpha}, \widetilde{\beta}, \widetilde{\gamma})\|_{\mathcal{K}}
\end{array}
$$

for all $2<q<4$, with $C$ depending on $\left\|\left(\widetilde{v}_{1}, \widetilde{\tau}_{1}, \widetilde{\alpha}_{1}, \widetilde{\beta}_{1}, \widetilde{\gamma}_{1}\right)\right\|_{\mathcal{K}}$ and $\left\|\left(\widetilde{v}_{2}, \widetilde{\tau}_{2}, \widetilde{\alpha}_{2}, \widetilde{\beta}_{2}, \widetilde{\gamma}_{2}\right)\right\|_{\mathcal{K}}$.

By adding the last four inequalities and taking $\varepsilon>0$ small enough, we have for all $2<q<4$ that

$$
\begin{gathered}
\|\tau\|_{W_{q}^{2,1}(Q)}+\|\alpha\|_{W_{q}^{2,1}(Q)}+\|\beta\|_{W_{q}^{2,1}(Q)}+\|\gamma\|_{W_{q}^{2,1}(Q)} \\
\leq C\|\tau\|_{L^{2}(Q)}+C\|\alpha\|_{L^{2}(Q)}+C\|\beta\|_{L^{2}(Q)}+C\|\gamma\|_{L^{2}(Q)}+C\|(\widetilde{v}, \widetilde{\tau}, \widetilde{\alpha}, \widetilde{\beta}, \widetilde{\gamma})\|_{\mathcal{K}},
\end{gathered}
$$

with $C$ depending on $\left\|\left(\widetilde{v}_{1}, \widetilde{\tau}_{1}, \widetilde{\alpha}_{1}, \widetilde{\beta}_{1}, \widetilde{\gamma}_{1}\right)\right\|_{\mathcal{K}}$ and $\left\|\left(\widetilde{v}_{2}, \widetilde{\tau}_{2}, \widetilde{\alpha}_{2}, \widetilde{\beta}_{2}, \widetilde{\gamma}_{2}\right)\right\|_{\mathcal{K}}$.

By multiplying inequality (3.6) by $\alpha$, integrating in $\Omega$ and using Hölder's and Young's inequalities, and also using that $\left(\tau_{1}, \alpha_{1}, \beta_{1}, \gamma_{1}\right),\left(\tau_{2}, \alpha_{2}, \beta_{2}, \gamma_{2}\right) \in W_{q}^{2,1}(Q) \subset$ $L^{\infty}(Q)$ (because $2<q<4$ ), we obtain

$$
\begin{aligned}
& \frac{1}{2} \frac{d}{d t} \int_{\Omega} \alpha^{2} d x+k \int_{\Omega}|\nabla \alpha|^{2} d x \\
\leq & -\int_{\Omega}\left(v_{2} \cdot \nabla \alpha\right) \alpha d x-\int_{\Omega}\left(v \cdot \nabla \alpha_{1}\right) \alpha d x+\int_{\Omega}\left(\alpha^{2}+\beta^{2}+\gamma^{2}+\tau^{2}\right) d x,
\end{aligned}
$$

where $C$ depends on $\left(\tau_{1}, \alpha_{1}, \beta_{1}, \gamma_{1}\right)$ and $\left(\tau_{2}, \alpha_{2}, \beta_{2}, \gamma_{2}\right)$.

Now, since $v \in V$ a.e. in $[0, T]$, we have that $\int_{0}^{t} \int_{\Omega}(v . \nabla \alpha) \alpha d x d t=0$ and, by using Hölder's, Gagliardo-Nirenberg's, and Young's inequalities, and estimate (3.5), we have

$$
\begin{aligned}
& \int_{\Omega}\left(v \cdot \nabla \alpha_{1}\right) \alpha d x \leq\|v\|_{L^{4}(\Omega)}\left\|\nabla \alpha_{1}\right\|_{L^{4}(\Omega)}\|\alpha\|_{L^{2}(\Omega)} \\
\leq & C\|v\|_{L^{4}(\Omega)}\|\nabla \tau\|_{L^{2}(\Omega)}^{1 / 2}\|\Delta \tau\|_{L^{2}(\Omega)}^{1 / 2}\|\alpha\|_{L^{4}(\Omega)} \leq C\|v\|_{L^{4}(\Omega)}\|\alpha\|_{L^{2}(\Omega)} \\
\leq & C\|v\|_{L^{4}(\Omega)}^{2}+C\|\alpha\|_{L^{2}(\Omega)}^{2} \leq C\|(\widetilde{v}, \widetilde{\tau}, \widetilde{\alpha}, \widetilde{\beta})\|_{\mathcal{K}}+C\|\alpha\|_{L^{2}(\Omega)}^{2} .
\end{aligned}
$$

Then,

$$
\frac{d}{d t} \int_{\Omega} \alpha^{2} d x \leq \int_{\Omega}\left(\alpha^{2}+\beta^{2}+\gamma^{2}+\tau^{2}\right) d x+C\|(\widetilde{v}, \widetilde{\tau}, \widetilde{\alpha}, \widetilde{\beta})\|_{\mathcal{K}}
$$

where $C$ depends on $\left(\tau_{1}, \alpha_{1}, \beta_{1}, \gamma_{1}, v_{1}\right)$ and $\left(\tau_{2}, \alpha_{2}, \beta_{2}, \gamma_{2}, v_{2}\right)$.

By proceeding similarly with the equations satisfied by $\beta, \gamma$, and $\tau$, we obtain

$$
\begin{aligned}
& \frac{d}{d t} \int_{\Omega} \beta^{2} d x \leq \int_{\Omega}\left(\alpha^{2}+\beta^{2}+\gamma^{2}+\tau^{2}\right) d x+C\|(\widetilde{v}, \widetilde{\tau}, \widetilde{\alpha}, \widetilde{\beta})\|_{\mathcal{K}}, \\
& \frac{d}{d t} \int_{\Omega} \gamma^{2} d x \leq \int_{\Omega}\left(\alpha^{2}+\beta^{2}+\gamma^{2}+\tau^{2}\right) d x+C\|(\widetilde{v}, \widetilde{\tau}, \widetilde{\alpha}, \widetilde{\beta})\|_{\mathcal{K}}, \\
& \frac{d}{d t} \int_{\Omega} \tau^{2} d x \leq C \int_{\Omega}\left(\alpha^{2}+\beta^{2}+\gamma^{2}+\tau^{2}\right) d x+C\|(\widetilde{v}, \widetilde{\tau}, \widetilde{\alpha}, \widetilde{\beta})\|_{\mathcal{K}},
\end{aligned}
$$

where $C$ depends on $\left(\tau_{1}, \alpha_{1}, \beta_{1}, \gamma_{1}, v_{1}\right)$ and $\left(\tau_{2}, \alpha_{2}, \beta_{2}, \gamma_{2}, v_{2}\right)$. 
By adding the last four equation and using Gronwall's Lemma,

$$
\int_{\Omega}\left(\alpha(t)^{2}+\beta(t)^{2}+\gamma(t)^{2}+\tau(t)^{2}\right) d x \leq C\|(\widetilde{v}, \widetilde{\tau}, \widetilde{\alpha}, \widetilde{\beta})\|_{\mathcal{K}}
$$

By replacing the last inequality in estimate (3.7), we get

$$
\|\tau\|_{W_{q}^{2,1}(Q)}+\|\alpha\|_{W_{q}^{2,1}(Q)}+\|\beta\|_{W_{q}^{2,1}(Q)}+\|\gamma\|_{W_{q}^{2,1}(Q)} \leq C\|(\widetilde{v}, \widetilde{\tau}, \widetilde{\alpha}, \widetilde{\beta}, \widetilde{\gamma})\|_{\mathcal{K}}
$$

for all $2<q<4$, with $C$ depending on $\left(\widetilde{v}_{1}, \widetilde{\tau}_{1}, \widetilde{\alpha}_{1}, \widetilde{\beta}_{1}, \widetilde{\gamma}_{1}\right)$ and $\left(\widetilde{v}_{2}, \widetilde{\tau}_{2}, \widetilde{\alpha}_{2}, \widetilde{\beta}_{2}, \widetilde{\gamma}_{2}\right)$.

Hence, $\mathcal{S}_{\lambda}: \mathcal{K} \rightarrow\left[L^{2}(0, T ; V) \cap L^{\infty}(0, T ; H)\right] \times\left[W_{q}^{2,1}(Q)\right]^{4}$ is continuous. Since $\left[L^{2}(0, T ; V) \cap L^{\infty}(0, T ; H)\right] \times\left[W_{q}^{2,1}(Q)\right]^{4} \subset \mathcal{K}$, with continuous imbedding, we get that $\mathcal{S}_{\lambda}: \mathcal{K} \rightarrow \mathcal{K}$ is continuous.

To prove that $\mathcal{S}_{\lambda}: \mathcal{K} \rightarrow \mathcal{K}$ is compact, let us show that $\mathcal{S}_{\lambda}: \mathcal{K} \rightarrow\left\{w \in L^{2}(0, T ; V)\right.$ : $\left.w_{t} \in L^{2}\left(0, T ; H^{\prime}\right)\right] \times\left[W_{q}^{2,1}(Q)\right]^{4}$ is bounded.

For this, let $\mathcal{A} \subset \mathcal{K}$ bounded, $(\widetilde{v}, \widetilde{\tau}, \widetilde{\alpha}, \widetilde{\beta}, \widetilde{\gamma}) \in \mathcal{K}$ arbitrary and $(v, \tau, \alpha, \beta, \gamma)=$ $\mathcal{S}_{\lambda}(\widetilde{v}, \widetilde{\tau}, \widetilde{\alpha}, \widetilde{\beta}, \widetilde{\gamma})$. By our previous estimates, we can conclude that the operator $\mathcal{S}_{\lambda}: \mathcal{K} \rightarrow\left[L^{2}(0, T ; V) \cap L^{\infty}(0, T ; H)\right] \times\left[W_{q}^{2,1}(Q)\right]^{4}$ is bounded. Next, by proceeding similarly as we did when we obtained estimates (3.4) and (3.5), we get that

$$
\begin{aligned}
\|v\|_{L^{4}(Q)} & \leq C\left(\|v\|_{L^{\infty}(0, T ; H)}+\|v\|_{\left.L^{2}(0, T ; V)\right)}\right) \\
& \leq C\left(\left\|v_{0}\right\|_{H}+\|(\widetilde{v}, \widetilde{\tau}, \widetilde{\alpha}, \widetilde{\beta}, \widetilde{\gamma})\|_{\mathcal{K}}+\|F\|_{L^{2}(Q)}\right) \leq C .
\end{aligned}
$$

By Lemma 3.2, we know the regularity of $v_{t}$; to obtain an estimate, we use the first equation of problem (3.3) and Hölder's and Young's inequalities to get

$$
\begin{gathered}
\left(v_{t}, u\right) \leq C\left(\|\nabla v\|_{L^{2}(\Omega)}+\|v\|_{L^{4}(\Omega)}^{2}+\|v\|_{L^{2}(\Omega)}+\|\widetilde{\tau}\|_{L^{2}(\Omega)}+\|\widetilde{\alpha}\|_{L^{2}(\Omega)}\right. \\
\left.+\|\widetilde{\beta}\|_{L^{2}(\Omega)}+\|\widetilde{\gamma}\|_{L^{2}(\Omega)}+\|F\|_{L^{2}(\Omega)}\right)\|u\|_{H_{0}^{1}(\Omega)}
\end{gathered}
$$

for all $u \in V, t \in(0, T)$. Thus,

$$
\begin{aligned}
\left\|v_{t}\right\|_{V^{\prime}} \leq C\left(\|v\|_{V}+\right. & \|v\|_{L^{4}(\Omega)}^{2}+\|\widetilde{\tau}\|_{L^{2}(\Omega)}+\|\widetilde{\alpha}\|_{L^{2}(\Omega)}+\|\widetilde{\beta}\|_{L^{2}(\Omega)} \\
& \left.+\|\widetilde{\gamma}\|_{L^{2}(\Omega)}+\|F\|_{L^{2}(\Omega)}\right),
\end{aligned}
$$

for all $t \in(0, T)$. From estimates (3.8), we conclude that

$$
\left\|v_{t}\right\|_{L^{2}\left(0, T ; V^{\prime}\right)} \leq C\left(\left\|v_{0}\right\|_{H}+\|(v, \widetilde{\tau}, \widetilde{\alpha}, \widetilde{\beta}, \widetilde{\gamma})\|_{\mathcal{K}}+\|F\|_{L^{2}(Q)}\right) \leq C .
$$

Then, $\mathcal{S}_{\lambda}: \mathcal{K} \rightarrow\left\{w \in L^{2}(0, T ; V): w_{t} \in L^{2}\left(0, T ; H^{\prime}\right)\right\} \times\left[W_{q}^{2,1}(Q)\right]^{4}$ is bounded. By using the compact imbedding $\left\{w \in L^{2}(0, T ; V): w_{t} \in L^{2}\left(0, T ; H^{\prime}\right)\right\} \subset L^{2}(0, T ; H)$ and Lemma 2.2, we get that $\mathcal{S}_{\lambda}: \mathcal{K} \rightarrow \mathcal{K}$ is compact for each fixed $\lambda \in[0,1]$.

Since $\lambda \in[0,1]$ is arbitrary, we conclude that $\mathcal{S}_{\lambda}: \mathcal{K} \rightarrow \mathcal{K}$ is continuous and compact for each fixed $\lambda \in[0,1]$.

Lemma 3.4. Assume that hypotheses (2.3), (2.4), and (2.5) hold. Let $f \in L^{q}(Q)$, with $2<q<4$. Given any bounded subset $\mathcal{A} \subset \mathcal{K}$, then $\mathcal{S}_{\lambda}(\widetilde{v}, \widetilde{\tau}, \widetilde{\alpha}, \widetilde{\beta}, \widetilde{\gamma})$ is continuous in $\lambda$, uniformly with respect to $(\widetilde{v}, \widetilde{\tau}, \widetilde{\alpha}, \widetilde{\beta}, \widetilde{\gamma}) \in \mathcal{A}$. 
Proof. Indeed, take $(\widetilde{v}, \widetilde{\tau}, \widetilde{\alpha}, \widetilde{\beta}, \widetilde{\gamma}) \in \mathcal{A}$ and consider $\lambda_{1}, \lambda_{2} \in[0,1]$ and $\left(v_{i}, \tau_{i}, \alpha_{i}, \beta_{i}, \gamma_{i}\right)=\mathcal{S}_{\lambda_{i}}(\widetilde{v}, \widetilde{\tau}, \widetilde{\alpha}, \widetilde{\beta}, \widetilde{\gamma})$, for $i=1,2$. Proceeding as before, we have

$$
\begin{aligned}
\left\|v_{1}-v_{2}\right\|_{L^{4}(Q)}+\| & \tau_{1}-\tau_{2}\left\|_{W_{q}^{2,1}(Q)}+\right\| \alpha_{1}-\alpha_{2} \|_{W_{q}^{2,1}(Q)} \\
& +\left\|\beta_{1}-\beta_{2}\right\|_{W_{q}^{2,1}(Q)}+\left\|\gamma_{1}-\gamma_{2}\right\|_{W_{q}^{2,1}(Q)} \leq C\left|\lambda_{1}-\lambda_{2}\right|,
\end{aligned}
$$

where $C$ depends on $\left\|\left(\widetilde{v}_{1}, \widetilde{\tau}_{1}, \widetilde{\alpha}_{1}, \widetilde{\beta}_{1}, \widetilde{\gamma}_{1}\right)\right\|_{\mathcal{K}}$ and $\left\|\left(\widetilde{v}_{2}, \widetilde{\gamma}_{2}, \widetilde{\alpha}_{2}, \widetilde{\beta}_{2}, \widetilde{\gamma}_{2}\right)\right\|_{\mathcal{K}}$.

From this, since $\mathcal{A}$ is bounded in $\mathcal{K}$, we obtain

$$
\left\|\left(v_{1}, \tau_{1}, \alpha_{1}, \beta_{1}, \gamma_{1}\right)-\left(v_{2}, \tau_{2}, \alpha_{2}, \beta_{2}, \gamma_{2}\right)\right\|_{\mathcal{K}} \leq C\left|\lambda_{1}-\lambda_{2}\right|,
$$

with $C$ depending only on $\mathcal{A}$, and thus $\mathcal{S}_{(\cdot)}(\widetilde{v}, \widetilde{\tau}, \widetilde{\alpha}, \widetilde{\beta}, \widetilde{\gamma}):[0,1] \rightarrow \mathcal{K}$ is continuous in $\lambda$, uniformly with respect to $(\widetilde{v}, \widetilde{\tau}, \widetilde{\alpha}, \widetilde{\beta}, \widetilde{\gamma}) \in \mathcal{A}$.

Lemma 3.5. Assume that hypotheses (2.3), (2.4), and (2.5) hold. Let $f \in L^{q}(Q)$, with $2<q<4$. Then, the operator $\mathcal{S}_{0}$ has a unique fixed point.

Proof. For $\lambda=0$ problem (3.3) becomes

$$
\begin{array}{lr}
\tau_{t}-b \Delta \tau+v \cdot \nabla \tau=l_{1} \alpha_{t}+l_{2} \beta_{t}+l_{3} \gamma_{t}+f, & \text { in } Q, \\
\alpha_{t}-k \Delta \alpha+v \cdot \nabla \alpha=g_{1}(\tau, \alpha, \beta, \gamma)+g_{3}(\tau, \alpha, \beta, \gamma), & \text { in } Q, \\
\beta_{t}-k \Delta \beta+v \cdot \nabla \beta=g_{2}(\tau, \alpha, \beta, \gamma)-g_{3}(\tau, \alpha, \beta, \gamma), & \text { in } Q, \\
\gamma_{t}-k \Delta \gamma+v \cdot \nabla \gamma=-g_{1}(\tau, \alpha, \beta, \gamma)-g_{2}(\tau, \alpha, \beta, \gamma), & \text { in } Q, \\
\frac{d}{d t}(v, u)-\nu(\nabla v, \nabla u)+(v \cdot \nabla v, u)=0, & \text { for all } u \in V, t \in(0, T), \\
\partial \tau / \partial n=\partial \alpha / \partial n=\partial \beta / \partial n=\partial \gamma / \partial n=0, & \text { on } \partial \Omega \times(0, T), \\
\tau=\tau_{0}, \alpha=\alpha_{0}, \beta=\beta_{0}, & \text { in } \Omega \times\{t=0\},
\end{array}
$$

where $g_{1}, g_{2}$, and $g_{3}$ are given by (1.2).

From theorems 3.1 and 3.2 in (Temam 1979), pp. 282 and 294, we conclude that there exists a unique $v \in L^{\infty}(0, T ; H) \cap L^{2}(0, T ; V) \subset L^{4}(Q)$, with $v_{t} \in L^{2}\left(0, T ; V^{\prime}\right)$, which is a unique solution of the fifth equation of the above problem; by applying Proposition 2.5 to the first four equations, we obtain that there exists a unique $(\tau, \alpha, \beta, \gamma) \in W_{q}^{2,1}(Q) \subset L^{\infty}(Q)$, which is a solution of the equations in the proposition for $q>2$. Thus, there exists a unique $(v, \tau, \alpha, \beta, \gamma) \in\left[L^{\infty}(0, T ; H) \cap L^{2}(0, T ; V)\right] \times$ $\left[W_{q}^{2,1}(Q)\right]^{4} \subset \mathcal{K}$, which solves the problem (3.3) with $\lambda=0$. Then, operator $\mathcal{S}_{0}$ has a unique fixed point $(v, \tau, \alpha, \beta, \gamma) \in \mathcal{K}$.

Lemma 3.6. Assume that hypotheses (2.3), (2.4), and (2.5) hold. Let $f \in L^{q}(Q)$, with $2<q<4$. There exists a constant $K>0$ independent of $\lambda$ so that every possible fixed point $(v, \tau, \alpha, \beta, \gamma)$ of $\mathcal{S}_{\lambda}$ satisfies $\|(v, \tau, \alpha, \beta, \gamma)\|_{\mathcal{K}} \leq K$ for all $\lambda \in[0,1]$.

Proof. For this, consider a fixed point $(v, \tau, \alpha, \beta, \gamma) \in \mathcal{K}$ of $\mathcal{S}_{\lambda}$ for some $\lambda \in[0,1]$, i.e. $(v, \tau, \alpha, \beta, \gamma)=\mathcal{S}_{\lambda}(v, \tau, \alpha, \beta, \gamma)$. Then, using the previous notations, $(v, \tau, \alpha, \beta, \gamma)$ 
satisfies

$$
\begin{array}{lc}
\tau_{t}-b \Delta \tau+v \cdot \nabla \tau=l_{1} \alpha_{t}+l_{2} \beta_{t}+l_{3} \gamma_{t}+f, & \text { in } Q, \\
\alpha_{t}-k \Delta \alpha+v \cdot \nabla \alpha=g_{1}(\tau, \alpha, \beta, \gamma)+g_{3}(\tau, \alpha, \beta, \gamma), & \text { in } Q, \\
\beta_{t}-k \Delta \beta+v \cdot \nabla \beta=g_{2}(\tau, \alpha, \beta, \gamma)-g_{3}(\tau, \alpha, \beta, \gamma), & \text { in } Q, \\
\gamma_{t}-k \Delta \gamma+v \cdot \nabla \gamma=-g_{1}(\tau, \alpha, \beta, \gamma)-g_{2}(\tau, \alpha, \beta, \gamma), & \text { in } Q, \\
\frac{d}{d t}(v, u)-\nu(\nabla v, \nabla u)+(v \cdot \nabla v, u)+\lambda(h(\alpha+\beta-\delta) v, u) \\
=\lambda(\mathcal{F}(\tau, \alpha, \beta, \gamma)+F, u) \quad \text { for all } u \in V, t \in(0, T), \\
\partial \tau / \partial n=\partial \alpha / \partial n=\partial \beta / \partial n=\partial \gamma / \partial n=0, & \text { on } \partial \Omega \times(0, T), \\
\tau=\tau_{0}, \alpha=\alpha_{0}, \beta=\beta_{0}, & \text { in } \Omega \times\{t=0\},
\end{array}
$$

where $g_{1}, g_{2}, g_{3}$, and $\mathcal{F}$ are given by (1.2) and (1.3).

First of all observe that by Lemma 3.2, when $(v, \tau, \alpha, \beta, \gamma)$ satisfies the previous problem, then $\alpha, \beta, \gamma \geq 0$ and $\alpha+\beta+\gamma=1$.

By Remark 2.6 we have

$$
\begin{aligned}
& \int_{\Omega}\left(\tau^{2}(t)+\alpha^{2}(t)+\beta^{2}(t)+\gamma^{2}(t)\right) d x+\int_{0}^{t} \int_{\Omega}\left(|\nabla \tau|^{2}+|\nabla \alpha|^{2}+|\nabla \beta|^{2}+|\nabla \gamma|^{2}\right) d x d t \\
\leq & C\left(\left\|\tau_{0}\right\|_{L^{2}(\Omega)}^{2}+\left\|\alpha_{0}\right\|_{L^{2}(\Omega)}^{2}+\left\|\beta_{0}\right\|_{L^{2}(\Omega)}^{2}+\|f\|_{L^{2}(Q)}^{2}\right)+C \int_{0}^{t} \int_{\Omega}|v|^{2} d x d t
\end{aligned}
$$

for all $0 \leq t \leq T$, where the constant $C$ depends on $\Omega, T$, and the constants of problem (3.9).

By replacing $u=v(t)$ in the last equation of problem (3.9) and integrating in $(0, t)$ with $0 \leq t \leq T$, we get

$$
\begin{aligned}
& \int_{\Omega}|v(t)|^{2} d x+\int_{0}^{t} \int_{\Omega}|\nabla v|^{2} d x d t+\int_{0}^{t} \int_{\Omega}(v \cdot \nabla v) v d x d t+\int_{0}^{t} \int_{\Omega} h(\alpha+\beta-\delta)|v|^{2} d x d t \\
= & \left\|v_{0}\right\|_{L^{2}(\Omega)}^{2}+\int_{0}^{t}(A \tau+B \alpha+C \beta+D \gamma+F, v) d t \\
\leq & \left\|v_{0}\right\|_{L^{2}(\Omega)}^{2}+C \int_{0}^{t} \int_{\Omega}\left(|v|^{2}+\tau^{2}+\alpha^{2}+\beta^{2}+F^{2}\right) d x d t .
\end{aligned}
$$

Next, we observe that $0 \leq \alpha+\beta \leq 1,0<\delta<\varepsilon$ and $h \geq 0$ in $(-\varepsilon, 1)$, so that $h(\alpha+$ $\beta-\delta) \geq 0$ in $Q$, and since $\int_{0}^{t} \int_{\Omega}(v \cdot \nabla v) v d x d t=0$, we have that

$$
\begin{aligned}
& \int_{\Omega}|v(t)|^{2} d x+\int_{0}^{t} \int_{\Omega}|\nabla v|^{2} d x d t \\
\leq & \left\|v_{0}\right\|_{L^{2}(\Omega)}^{2}+C \int_{0}^{t} \int_{\Omega}\left(|v|^{2}+\tau^{2}+\alpha^{2}+\beta^{2}+F^{2}\right) d x d t .
\end{aligned}
$$

By adding the last inequality to the inequality (3.10) and then applying Gronwall's lemma, it follows that

$$
\int_{\Omega}\left(|v(t)|^{2}+\tau^{2}(t)+\alpha^{2}(t)+\beta^{2}(t)+\gamma^{2}(t)\right) d x
$$




$$
\begin{aligned}
& +\int_{0}^{t} \int_{\Omega}\left(|\nabla v|^{2}+|\nabla \tau|^{2}+|\nabla \alpha|^{2}+|\nabla \beta|^{2}+|\nabla \gamma|^{2}\right) d x d t \\
& \leq C\left(\left\|v_{0}\right\|_{L^{2}(\Omega)}^{2}+\left\|\tau_{0}\right\|_{L^{2}(\Omega)}^{2}+\left\|\alpha_{0}\right\|_{L^{2}(\Omega)}^{2}+\left\|\beta_{0}\right\|_{L^{2}(\Omega)}^{2}+\left\|\gamma_{0}\right\|_{L^{2}(\Omega)}^{2}\right. \\
& \left.+\|f\|_{L^{2}(Q)}^{2}+\|F\|_{L^{2}(Q)}^{2}\right)
\end{aligned}
$$

for all $0 \leq t \leq T$.

From this, we get

$$
\begin{array}{r}
\|v\|_{L^{\infty}(0, T ; H)}+\|v\|_{L^{2}(0, T ; V)} \leq C\left(\left\|v_{0}\right\|_{L^{2}(\Omega)}+\left\|\tau_{0}\right\|_{L^{2}(\Omega)}+\left\|\alpha_{0}\right\|_{L^{2}(\Omega)}\right. \\
\left.+\left\|\beta_{0}\right\|_{L^{2}(\Omega)}+\left\|\gamma_{0}\right\|_{L^{2}(\Omega)}+\|f\|_{L^{2}(Q)}+\|F\|_{L^{2}(Q)}\right),
\end{array}
$$

and since $\|v\|_{L^{4}(Q)} \leq C\|v\|_{L^{\infty}(0, T ; H)}^{1 / 2}\|v\|_{L^{2}(0, T ; V)}^{1 / 2}$, it follows that

$$
\begin{gathered}
\|v\|_{L^{4}(Q)} \leq C\left(\left\|v_{0}\right\|_{L^{2}(\Omega)}+\left\|\tau_{0}\right\|_{L^{2}(\Omega)}+\left\|\alpha_{0}\right\|_{L^{2}(\Omega)}+\left\|\beta_{0}\right\|_{L^{2}(\Omega)}+\left\|\gamma_{0}\right\|_{L^{2}(\Omega)}\right. \\
\left.+\|f\|_{L^{2}(Q)}+\|F\|_{L^{2}(Q)}\right) .
\end{gathered}
$$

Now, by Proposition 2.5,

$$
\begin{aligned}
& \|\tau\|_{W_{q}^{2,1}(Q)}+\|\alpha\|_{W_{q}^{2,1}(Q)}+\|\beta\|_{W_{q}^{2,1}(Q)}+\|\gamma\|_{W_{q}^{2,1}(Q)} \\
\leq & C\left(\left\|\tau_{0}\right\|_{W_{2}^{2}(\Omega)}+\left\|\alpha_{0}\right\|_{W_{2}^{2}(\Omega)}+\left\|\beta_{0}\right\|_{W_{2}^{2}(\Omega)}+\left\|\gamma_{0}\right\|_{W_{2}^{2}(\Omega)}+\|f\|_{L^{q}(Q)}\right),
\end{aligned}
$$

with the constant $C$ depending on $\Omega, T,\|v\|_{L^{4}(Q)}$, and the constants of Problem (3.9). By (3.13), we conclude that

$$
\begin{aligned}
& \|\tau\|_{W_{q}^{2,1}(Q)}+\|\alpha\|_{W_{q}^{2,1}(Q)}+\|\beta\|_{W_{q}^{2,1}(Q)}+\|\gamma\|_{W_{q}^{2,1}(Q)} \\
\leq & C\left(\left\|\tau_{0}\right\|_{W_{2}^{2}(\Omega)}+\left\|\alpha_{0}\right\|_{W_{2}^{2}(\Omega)}+\left\|\beta_{0}\right\|_{W_{2}^{2}(\Omega)}+\left\|\gamma_{0}\right\|_{W_{2}^{2}(\Omega)}+\|f\|_{L^{q}(Q)}\right),
\end{aligned}
$$

with the constant $C$ depending on $\Omega, T$, the constants and parameters of Problem (3.9), $\left\|v_{0}\right\|_{L^{2}(\Omega)},\left\|\tau_{0}\right\|_{L^{2}(\Omega)},\left\|\alpha_{0}\right\|_{L^{2}(\Omega)}\left\|\beta_{0}\right\|_{L^{2}(\Omega)},\left\|\gamma_{0}\right\|_{L^{2}(\Omega)},\|f\|_{L^{2}(Q)}$, and $\|F\|_{L^{2}(Q)}$.

Since for $2<q<4, W_{q}^{2,1}(Q) \subset L^{\infty}(Q)$ with continuous imbedding, we have

$$
\begin{aligned}
& \|(v, \tau, \alpha, \beta, \gamma)\|_{\mathcal{K}} \\
\leq & C\left(\|v\|_{L^{4}(Q)}+\|\tau\|_{W_{q}^{2,1}(Q)}+\|\alpha\|_{W_{q}^{2,1}(Q)}+\|\beta\|_{W_{q}^{2,1}(Q)}+\|\gamma\|_{W_{q}^{2,1}(Q)}\right) \\
\leq & C\left(\left\|v_{0}\right\|_{L^{2}(\Omega)}+\left\|\tau_{0}\right\|_{W_{2}^{2}(\Omega)}+\left\|\alpha_{0}\right\|_{W_{2}^{2}(\Omega)}+\left\|\beta_{0}\right\|_{W_{2}^{2}(\Omega)}+\left\|\gamma_{0}\right\|_{W_{2}^{2}(\Omega)}\right. \\
& \left.\quad+\|f\|_{L^{q}(Q)}+\|F\|_{L^{2}(Q)}\right)
\end{aligned}
$$

where $C$ does not depend on $\lambda \in[0,1]$.

Finally, we can use the previous lemmas to prove Proposition 3.1.

\section{Proof of Proposition 3.1.}

Proof. By lemmas 3.2 through 3.6 we obtain that the hypotheses of LeraySchauder's fixed point theorem are satisfied. By applying the mentioned theorem we conclude that there exists $(v, \tau, \alpha, \beta, \gamma) \in\left\{w \in L^{\infty}(0, T ; H) \cap L^{2}(0, T ; V): w_{t} \in\right.$ $\left.L^{2}\left(0, T ; V^{\prime}\right)\right\} \times\left[W_{q}^{2,1}(Q)\right]^{4} \subset \mathcal{K}$, which is a fixed point of $\mathcal{S}_{1}$; that is, there exists a solution of problem (3.1)-(3.2). From inequalities (3.12) and (3.14), we obtain the estimate of Proposition (3.1). 


\section{Passage to the limit}

In this section we will pass to the limit as $\delta \rightarrow 0$ along the solutions of the family of problem (3.1)-(3.2), which were obtained in Proposition 3.1.

For this, with the notation of the beginning of the previous section, we summarize our results in the following.

Lemma 4.1. Assume that hypotheses (2.3), (2.5), and (2.4) hold. There exists a constant $C$, independent of $\delta \in(0, \varepsilon)$, such that any solution $\left(v^{\delta}, \tau^{\delta}, \alpha^{\delta}, \beta^{\delta}\right)$ of the regularized problem (3.1)-(3.2) satisfies

1. $\left\|v^{\delta}\right\|_{L^{\infty}(0, T ; H) \cap L^{2}(0, T ; V)}+\int_{0}^{T} \int_{\Omega} h\left(\alpha^{\delta}+\beta^{\delta}-\delta\right)\left|v^{\delta}\right|^{2} d x d t \leq C ;$

2. $\left\|\tau^{\delta}\right\|_{L^{\infty}\left(0, T ; L^{2}(\Omega)\right) \cap L^{2}\left(0, T ; H_{0}^{1}(\Omega)\right)} \leq C$;

3. $\left\|\alpha^{\delta}\right\|_{L^{\infty}\left(0, T ; L^{2}(\Omega)\right) \cap L^{2}\left(0, T ; H_{0}^{1}(\Omega)\right)} \leq C$;

4. $\left\|\beta^{\delta}\right\|_{L^{\infty}\left(0, T ; L^{2}(\Omega)\right) \cap L^{2}\left(0, T ; H_{0}^{1}(\Omega)\right)} \leq C$;

5. $\left\|\gamma^{\delta}\right\|_{L^{\infty}\left(0, T ; L^{2}(\Omega)\right) \cap L^{2}\left(0, T ; H_{0}^{1}(\Omega)\right)} \leq C$;

6. $\left\|\tau^{\delta}\right\|_{W_{q}^{2,1}(Q)},\left\|\alpha^{\delta}\right\|_{W_{q}^{2,1}(Q)},\left\|\beta^{\delta}\right\|_{W_{q}^{2,1}(Q)},\left\|\gamma^{\delta}\right\|_{W_{q}^{2,1}(Q)} \leq C$, for $2 \leq q<4$.

As consequence, we have the following.

Lemma 4.2. Assume that hypotheses (2.3), (2.4), and (2.5) hold, and let $f \in L^{q}(Q)$, with $2<q<4$. There exist $\tau, \alpha, \beta, \gamma \in W_{q}^{2,1}(Q) \cap C\left(0, T ;\left(H^{1}(\Omega)\right)^{\prime}\right), v \in L^{\infty}(0, T ; H) \cap$ $L^{2}(0, T ; V)$ and subsequences of the solutions $\left(v^{\delta}, \tau^{\delta}, \alpha^{\delta}, \beta^{\delta}, \gamma^{\delta}\right)$ of the regularized problem (3.1)-(3.2), which for simplicity of notation we do not change the superscripts, such that

$\tau^{\delta} \rightarrow \tau \quad$ in $L^{q}(Q) \cap C\left(0, T ;\left(H^{1}(\Omega)\right)^{\prime}\right)$ strongly,

$\tau^{\delta} \rightarrow \tau \quad$ in $L^{2}\left(0, T ; H^{1}(\Omega)\right)$ weakly

$\tau_{t}^{\delta} \rightarrow \tau_{t}$ in $L^{q}(Q)$ weakly

$\alpha^{\delta} \rightarrow \alpha \quad$ in $L^{q}\left(0, T ; W_{q}^{1}(\Omega)\right) \cap C\left(0, T ;\left(H^{1}(\Omega)\right)^{\prime}\right)$ strongly,

$\alpha^{\delta} \rightarrow \alpha$ in $L^{2}\left(0, T ; H^{1}(\Omega)\right)$ weakly,

$\alpha_{t}^{\delta} \rightarrow \alpha_{t}$ in $L^{q}(Q)$ weakly,

$\beta^{\delta} \rightarrow \beta$ in $L^{q}\left(0, T ; W_{q}^{1}(\Omega)\right) \cap C\left(0, T ;\left(H^{1}(\Omega)\right)^{\prime}\right)$ strongly,

$\beta^{\delta} \rightarrow \beta$ in $L^{2}\left(0, T ; H^{1}(\Omega)\right)$ weakly,

$\beta_{t}^{\delta} \rightarrow \beta_{t}$ in $L^{q}(Q)$ weakly,

$\gamma^{\delta} \rightarrow \gamma \quad$ in $L^{q}\left(0, T ; W_{q}^{1}(\Omega)\right) \cap C\left(0, T ;\left(H^{1}(\Omega)\right)^{\prime}\right)$ strongly,

$\gamma^{\delta} \rightarrow \gamma \quad$ in $L^{2}\left(0, T ; H^{1}(\Omega)\right)$ weakly, and

$\gamma_{t}^{\delta} \rightarrow \gamma_{t}$ in $L^{q}(Q)$ weakly.

$\alpha^{\delta} \rightarrow \alpha, \beta^{\delta} \rightarrow \beta$, and $\gamma^{\delta} \rightarrow \gamma$ uniformly in $\bar{Q}$,

Moreover,

$$
\alpha \geq 0, \beta \geq 0, \gamma \geq 0 \text { and } \alpha+\beta+\gamma=1 \text { in } \bar{Q} .
$$


Also, there is $v \in L^{\infty}(0, T ; H) \cap L^{2}(0, T ; V)$ and a subsequence such that

$$
\begin{aligned}
& v^{\delta} \rightarrow v \text { in } L^{2}(0, T ; V) \text { weakly, } \\
& v^{\delta} \stackrel{*}{\rightarrow} v \text { in } L^{\infty}(0, T ; H) \text { weakly star. }
\end{aligned}
$$

Proof. By using Lemma 4.1 and the compact embedding given in (Simon 1987, Corollary 4), we conclude that there exist $\tau, \alpha, \beta, \gamma \in W_{q}^{2,1}(Q) \cap C\left(0, T ;\left(H^{1}(\Omega)\right)^{\prime}\right) \subset$ $L^{2}\left(0, T ; H^{1}(\Omega)\right)$ and subsequences of $\left(\tau^{\delta}\right),\left(\alpha^{\delta}\right),\left(\beta^{\delta}\right),\left(\gamma^{\delta}\right)$, which for simplicity of notation we still denote by $\left(\tau^{\delta}\right),\left(\alpha^{\delta}\right),\left(\beta^{\delta}\right)$, and $\left(\gamma^{\delta}\right)$, such that the convergences in (4.1) hold.

Moreover, since $\alpha^{\delta}, \beta^{\delta}$, and $\gamma^{\delta}$ are bounded sequences in $W_{q}^{2,1}(Q)$, with $2<q<4$, and $W_{q}^{2,1}(Q) \subset H^{\lambda, \lambda / 2}(\bar{Q})$, with $\lambda=2-4 / q$ (see (Ladyzhenskaya 1968), pp. 80), and thus are uniformly bounded and equicontinuous sequences of functions on $\bar{Q}$. By the Arzelà-Ascoli theorem, we can also assume that the convergences in (4.2) are true, which obviously imply that $\alpha \geq 0, \beta \geq 0, \gamma \geq 0$, and $\alpha+\beta+\gamma=1$.

The known uniform estimates for $v^{\delta}$ in $L^{2}(0, T ; V)$ and $L^{\infty}(0, T ; H)$ immediately imply (4.3).

The just obtained functions $\alpha$ and $\beta$ can now to be used to define the following sets, as stated in Theorem 2.7:

$$
\begin{gathered}
\Omega_{m l}(0)=\left\{x \in \Omega: \alpha_{0}(x, t)+\beta_{0}(x, t)<1\right\}, \\
Q_{m l}=\{(x, t) \in Q: \alpha(x, t)+\beta(x, t)<1\}, \\
Q_{s}=\{(x, t) \in Q: \alpha(x, t)+\beta(x, t)=1\} .
\end{gathered}
$$

We also remark that since $\alpha$ and $\beta$ are continuous functions, $Q_{m l}$ is an open set.

Lemma 4.3. Assume that hypotheses (2.3), (2.5), and (2.4) hold. For any $0 \leq$ $t_{1}<t_{2} \leq T$ and $U \subset \Omega$ such that $\bar{U} \times\left[t_{1}, t_{2}\right] \subset Q_{m l} \cup \Omega_{m l}(0)$, there exist a constant $C$ and $\delta_{0} \in(0, \delta)$ such that for all $\delta \in\left(0, \delta_{0}\right)$, for any solution $\left(v^{\delta}, \tau^{\delta}, \alpha^{\delta}, \beta^{\delta}, \gamma^{\delta}\right)$ of the regularized problem (3.1)-(3.2), it holds that

$$
\left\|v_{t}^{\delta}\right\|_{L^{2}\left(t_{1}, t_{2} ; V(U)^{\prime}\right)} \leq C .
$$

Proof. Take any $0 \leq t_{1}<t_{2} \leq T$ and $U \subset \Omega$ such that $\bar{U} \times\left[t_{1}, t_{2}\right] \subset Q_{m l} \cup \Omega_{m l}(0)$, and fix $\delta \in(0, \varepsilon)$.

By the first equation of Problem (3.2), we have

$$
\begin{aligned}
<v_{t}^{\delta}, u>=-\nu \int_{U} & \nabla v^{\delta} \cdot \nabla u d x-\int_{U}\left(v^{\delta} \cdot \nabla v^{\delta}\right) u d x \\
& -\int_{U} h\left(\alpha^{\delta}+\beta^{\delta}-\delta\right) v^{\delta} u d x-\int_{U}\left(A \tau^{\delta}+B \alpha^{\delta}+C \beta^{\delta}+D \gamma^{\delta}+F\right) u d x
\end{aligned}
$$

for all $u \in V(U)$, a.e. in $\left[t_{1}, t_{2}\right]$.

Since $0 \leq \alpha+\beta<1$ in $Q_{m l} \cup \Omega_{m l}(0)$ and $\bar{U} \times\left[t_{1}, t_{2}\right] \subset Q_{m l} \cup \Omega_{m l}(0)$ is a compact set, there exists $\rho \in(0,1)$ such that $\alpha+\beta<1-\rho$ in $\left[t_{1}, t_{2}\right] \times \bar{U}$. Remembering that $\alpha^{\delta}+\beta^{\delta}$ converges uniformly to $\alpha+\beta$, it follows that there exists $\delta_{0} \in(0, \varepsilon)$ such that 
if $\delta \in\left(0, \delta_{0}\right)$ then $\alpha_{\delta}+\beta_{\delta}<1-\rho / 2$ in $\bar{U} \times\left[t_{1}, t_{2}\right]$. Thus, $0 \leq h\left(\alpha_{\delta}+\beta_{\delta}-\delta\right) \leq h(1-\rho / 2)$ in $Q_{m l} \cup \Omega_{m l}(0)$ for $\delta \in\left(0, \delta_{0}\right)$, because $h$ is a non-decreasing function.

Consequently, $h\left(\alpha_{\delta}+\beta_{\delta}-\delta\right)$ is uniformly bounded with respect to $\delta \in\left(0, \delta_{0}\right)$ in $L^{\infty}\left(U \times\left(t_{1}, t_{2}\right)\right)$.

Next, by applying Hölder's and Young's inequalities in (4.7) and using the previous results, we obtain

$$
\begin{aligned}
\left\|v_{t}^{\delta}\right\|_{L^{2}\left(t_{1}, t_{2} ; V^{\prime}(U)\right)} \leq C[ & \left\|v^{\delta}\right\|_{L^{2}\left(t_{1}, t_{2} ; V(U)\right)}+\left\|v^{\delta}\right\|_{L^{2}\left(t_{1}, t_{2} ; V(U)\right)}^{2} \\
& +\left\|h\left(\alpha^{\delta}+\beta^{\delta}-\delta\right)\right\|_{L^{\infty}\left(U \times\left(t_{1}, t_{2}\right)\right)}\left\|v^{\delta}\right\|_{L^{2}\left(t_{1}, t_{2} ; H(U)\right)}+\left\|\tau^{\delta}\right\|_{L^{2}(Q)} \\
& \left.+\left\|\alpha^{\delta}\right\|_{L^{2}(Q)}+\left\|\beta^{\delta}\right\|_{L^{2}(Q)}+\left\|\gamma^{\delta}\right\|_{L^{2}(Q)}+\|F\|_{L^{2}(Q)}\right] \\
\leq C &
\end{aligned}
$$

for all $\delta \in\left(0, \delta_{0}\right)$.

\section{Proof of Theorem 2.7}

Proof. We consider $v, \tau, \alpha, \beta, \gamma$, and the subsequences given in Lemma 4.2. Then, the convergences stated in Lemma 4.2 are enough to pass to the limit as $\delta \rightarrow 0+$ in (3.1) along a subsequence to give, in a standard way, that first four equations of problem (1.1) or (2.6) are satisfied by $(v, \tau, \alpha, \beta, \gamma)$.

To obtain seventh equation of Problem (2.6), we have to be more careful. Take any $0 \leq t_{1}<t_{2} \leq T$ and $U \subset \Omega$ such that $\bar{U} \times\left[t_{1}, t_{2}\right] \subset Q_{m l} \cup \Omega_{m l}(0)$; by Lemma $4.1,\left(v^{\delta}\right)$ is a bounded sequence in $L^{2}\left(t_{1}, t_{2} ; H^{1}(U)\right)$, and by Lemma $4.3,\left(v_{t}^{\delta}\right)$ is a bounded sequence $L^{2}\left(t_{1}, t_{2} ; V(U)^{\prime}\right)$, for all $0 \leq t_{1}<t_{2} \leq T$ and $U \subset \Omega$ such that $\left[t_{1}, t_{2}\right] \times \bar{U} \subset Q_{m l} \cup \Omega_{m l}(0)$. Then, by compact embedding (Simon 1987, Corollary 4) there exist $v$ and a subsequence of $\left(v^{\delta}\right)$ (which we still denote by $\left(v^{\delta}\right)$ ) such that $\left(v^{\delta}\right)$ converges strongly to $v$ in $L^{2}\left(\left(t_{1}, t_{2}\right) \times U\right)$.

Next, we observe that $Q_{m l}$ can be covered by a countable number of sets $U_{i} \times\left(t_{i}, t_{i+1}\right)$ such that $\overline{U_{i}} \times\left[t_{i}, t_{i+1}\right] \subset Q_{m l} \cup \Omega_{m l}(0)$; then by a diagonal argument, we obtain that along a suitable subsequence besides the previous convergences we also have

$$
v^{\delta} \rightarrow v \text { in } L_{l o c}^{2}\left(Q_{m l} \cup \Omega_{m l}(0)\right) .
$$

Then, by taking $u=\eta \in \mathcal{W}_{m l}$ in the first equation of Problem (3.2), after integrating over $[0, T]$ and doing an integration by parts in the term $\int_{0}^{t}\left(v_{t}^{\delta}, \eta\right) d s$ by using the properties of $\eta$, we find

$$
\begin{aligned}
-\int_{0}^{T} & <v^{\delta}, \eta_{t}>d s-\left(v^{\delta}(0), \eta(0)\right)_{\Omega_{m l}(0)} \\
& \quad+\int_{0}^{T}\left[\nu\left(\nabla v^{\delta}, \nabla \eta\right)+\left(v^{\delta} \cdot \nabla v^{\delta}, \eta\right)+\left(h\left(\alpha^{\delta}+\beta^{\delta}-\delta\right) v^{\delta}, \eta\right)\right] d s \\
= & \left.\int_{0}^{t}\left[\mathcal{F}\left(\tau^{\delta}, \alpha^{\delta}, \beta^{\delta}\right), \eta\right)+(F, \eta)\right] d s .
\end{aligned}
$$

Finally observe that by (4.2) we get $\alpha^{\delta}+\beta^{\delta}-\delta \rightarrow \alpha+\beta$ uniformly on compact subsets of $Q$. Besides, since $h \in C^{1}([0,1))$ and $0 \leq \alpha+\beta \leq 1$ in $Q_{m l}$, then $h\left(\alpha^{\delta}+\beta^{\delta}-\right.$ $\delta) \rightarrow h(\alpha+\beta)$ uniformly on compact subsets of $Q_{m l} \cup \Omega_{m l}(0)$.

This last convergence and (5.1)-(4.1) allow us to pass to the limit in (5.2) and obtain that $v$ satisfies the seventh equation in (2.6). 
To check that $v=0$ a.e. in $Q_{s}$ given by (4.6), take any compact set $K \subset Q_{s}$ and $\epsilon>0$ arbitrary. Since $K$ is compact, $0 \leq \alpha^{\delta}+\beta^{\delta} \leq 1$ and $\alpha^{\delta}+\beta^{\delta}$ converges to $\alpha+\beta=1$ uniformly on $K$, so there exist $\delta_{K} \in(0,1)$ such that $1-\epsilon \leq \alpha^{\delta}+\beta^{\delta} \leq 1$ in $K$ for $\delta \in\left(0, \delta_{K}\right)$ and consequently $h(1-\delta-\epsilon) \leq h\left(\alpha^{\delta}+\beta^{\delta}-\delta\right)$ in $K$ for $\delta \in\left(0, \delta_{K}\right)$. From the last inequality and Lemma 4.1 , it follows that

$$
h(1-\delta-\epsilon)\left\|v^{\delta}\right\|_{L^{2}(K)}^{2} \leq \int_{0}^{T} \int_{\Omega} h\left(\alpha^{\delta}+\beta^{\delta}\right)\left|v^{\delta}\right|^{2} d x d t \leq C
$$

for all $\delta \in\left(0, \delta_{K}\right)$.

By passing to the limit $\delta \rightarrow 0^{+}$in last inequality, we obtain that $h(1-\epsilon)\|v\|_{L^{2}(K)}^{2} \leq$ $C$, because $h \in C^{1}([0,1))$. Since $\epsilon>0$ is arbitrary and $\lim _{\theta \rightarrow 1^{-}} h(\theta)=+\infty$, by $(2.3)$, we then conclude that $\|v\|_{L^{2}(K)}^{2}=0$. Therefore $v=0$ a.e. in $K$, and since $K$ is an arbitrary compact subset of $Q_{s}$, we have that $v=0$ a.e. $Q_{s}$ as required in (2.6).

Hence, $(v, \tau, \alpha, \beta, \gamma)$ is a solution of Problem (2.6).

Acknowledgment. The first author was partially supported by FAPESP, Brazil, Grant 2010/10087-8. The second author was partially supported by CNPq, Brazil, Grant 307833/2008-9.

\section{REFERENCES}

[1] R. Adams, Sobolev Spaces, Academic Press, 1975.

[2] J.L. Boldrini, B.M.C. Caretta, and E. Fernández-Cara, Analysis of a two-phase field model for the solidification of an alloy, J. Math. Anal. Appl., 357, 25-44, 2009.

[3] J.L. Boldrini and G. Planas, Weak solutions of a phase-field model for phase-change of an alloy with thermal properties, Math. Meth. Appl. Sci., 25(14), 1177-1193, 2002.

[4] J.L. Boldrini and G. Planas, A tridimensional phase-field model with convection for phasechange of an alloy, Disc. Cont. Dyn. Syst., 13(2), 429-450, 2005.

[5] J.L. Boldrini and C.L.D. Vaz, Existence and regularity of solutions of a phase field model for solidification with convection of pure materials in two dimensions, Electron. J. Diff. Equ., 109, 1-25, 2003.

[6] G. Caginalp and J. Jones, A derivation and analysis of phase field models of thermal alloys, Annal. Phys., 237, 66-107, 1995.

[7] G. Caginalp, Phase field computations of single-needle crystals, crystal growth and motion by mean curvature, SIAM J. Sci. Comput., 15(1), 106-126, 1994.

[8] G. Caginalp, Stefan and Hele-Shaw type models as asymptotic limits of the phase-field equations, Phys. Rev. A, 39(11), 5887-5896, 1989.

[9] G. Caginalp, An analysis of phase field model of a free boundary, Arch. Ration. Mech. Anal., 92, 205-245, 1986.

[10] B.M.R. Calsavara and J.L. Boldrini, Solutions of an advected phase field system with low regularity velocity, Proc. Amer. Math. Soc., 141(3), 943-958.

[11] B.M.C. Caretta and J.L. Boldrini, Three-dimensional solidification with two possible crystallizations states: Existence of solutions with flow in the melt, Math. Meth. Appl. Sci., 33(5), 655-675, 2010.

[12] P. Colli and J. Sprekel, Stefan problems and the Penrose-Fife field model, Adv. Math. Sci. Appl., 7, 911-934, 1997.

[13] P. Colli and J. Sprekel, Weak solutions to some Penrose-Fife phase-field systems with dependent memory, J. Diff. Equ., 142(1), 54-77, 1998.

[14] P. Colli and J. Sprekel, Global solutions to the Penrose-Fife phase-field model with zero interfacial energy and Fourier Law, Adv. Math. Sci. Appl., 9(1), 383-391, 1999.

[15] A. Friedman, Partial Differential Equations of Parabolic Type, Prentice Hall, 1964.

[16] A. Friedman, Partial Differential Equations, Robert E. Krieger Publishing Company, New York, 1976

[17] K. Hoffman and L. Jiang, Optimal control of a phase field model for solidification, Numer. Funct. Anal. Optim., 13, 11-27, 1992.

[18] W. Horn, P. Laurençot, and J. Sprekel, Global solution to a Penrose-Fife phase-field model 
unde flux boundary conditions for the inverse temperature, Math. Meth. Appl. Sci., 19, 1053-1072, 1996.

[19] P. Krejči, J. Sprekel, and U. Stefanelli, Phase-field models with hysteresis in one dimensional thermo-visco-plasticity, SIAM J. Math. Anal., 34(2), 409-434, 2002.

[20] O.A. Ladyzenskaja, V.A. Solonnikov, and N.N. Ural'ceva, Linear and Quasilinear Equations of Parabolic Type, Translations of Mathematical Monographs, American Mathematical Society, Providence, Rhode Island, 23, 1968.

[21] J.L. Lions, Control of Distributed Singular Systems, Gauthier-Villars, 1985.

[22] V.P. Mikhaylov, Partial Differential Equations, Mir Publishers, Moscow, 1978.

[23] G. Planas and J.L. Boldrini, A bidimensional phase-field model with convection for change phase of an alloy, J. Math. Anal. Appl., 303, 669-687, 2005.

[24] G. Planas, Existence of solutions to a phase-field model with phase-dependent heat absorption, Electron. J. Diff. Equ., 28, 1-12, 2007.

[25] P. Sprekel and S. Zheng, Global existence and asymptotic behaviour for a nonlocal phase-field model for non-isothermal phase transitions, J. Math. Anal., 279, 97-110, 2003.

[26] J.P. Simon, Compact sets in the space $L^{p}(0, T ; B)$, Ann. Mat. Pura Appl., serie quarta, 146, 65-96, 1987.

[27] I. Steinbach, F. Pezzolla, B. Nestler, M. Seesselberg, R. Prieler, G.J. Schimitz, and J.L.L. Rezende, A phase field concept for multiphase systems, Phys. D, 94, 135-147, 1996.

[28] I. Steinbach and F. Pezzolla, A generalized field method for multiphase transformations using interface fields, Phys. D, 134, 385-393, 1999.

[29] R. Temam, Navier-Stokes Equations, Studies in Mathematics and its Applications, NorthHolland Publishing Company, 1979.

[30] C.L.D. Vaz and J.L. Boldrini, A semidiscretization scheme for a phase-field type model for solidification, Port. Math., 63(3), 261-292, 2006. 